\title{
Insights into the origin of the excited transitions in graphene quantum dots interacting with heavy metals in different media
}

Ivan Shtepliuk, Volodymyr Khranovskyy and Rositsa Yakimova

The self-archived postprint version of this journal article is available at Linköping University Institutional Repository (DiVA):

http:/ / urn.kb.se/ resolve?urn=urn:nbn:se:liu:diva- 143610

N.B.: When citing this work, cite the original publication.

Shtepliuk, I., Khranovskyy, V., Yakimova, R., (2017), Insights into the origin of the excited transitions in graphene quantum dots interacting with heavy metals in different media, Physical Chemistry,

Chemical Physics - PCCP, 19(45), 30445-30463. https:/ / doi.org/ 10.1039/ c7cp04711h

Original publication available at:

https:// doi.org/ 10.1039/c7cp04711h

Copyright: Royal Society of Chemistry

http:// www.rsc.org/ 


\title{
Insights into the origin of the excited transitions in graphene quantum dots interacting with heavy metals in different media
}

Ivan Shtepliuk, Volodymyr Khranovskyy and Rositsa Yakimova

\begin{abstract}
Department of Physics, Chemistry and Biology, Linköping University, SE-58183, Linköping,
\end{abstract}

\section{Sweden}

\begin{abstract}
Exploring graphene quantum dots (GQDs) is an attractive way to design novel optical and electrochemical sensors for fast and reliable detection of toxic heavy metals (HMs), such as $\mathrm{Cd}, \mathrm{Hg}$ and $\mathrm{Pb}$. There are two main strategies of achieving this: (i) surface modification of anelectrochemical working electrode by nanoscale GQDs and (ii) using a GQD solution electrolyte for optical sensing. Further development of these sensing technologies towards reaching or exceeding the WHO permissible limits implies deep understanding of the interaction between GQDs and HMs in different dielectric media. Solvent is expected to be one of the key factors affecting the binding ability of the GQDs to HMs and their electronic and optical properties. Here we show that the solvent-solute interaction changes the geometrical configuration, stability and absorption spectra of zigzag/armchair-edged GQDs after complexation with neutral and charged HM species. We observe physisorption behavior of $\mathrm{Cd}$ and $\mathrm{Hg}$ adatoms on the $s p^{2}$ surface with a solvent-mediated enhancement of the binding energy with increasing the solvent polarity. For Pb adatoms, an opposite picture is revealed. We find that the solvent effect also manifests itself in weakening of the chemisorption strength in HM cation- $\pi$ system with increasing the dielectric constant of the solvent. Thus, a solvent engineering strategy based on control of the dielectric permittivity can be a promising approach to reach the desired binding energy in the HM@GQDs and to provide high sensitivity and selectivity of both optical and electrochemical sensor to toxic HMs we are interested in.
\end{abstract}

Keywords: DFT, TD-DFT, graphene quantum dots, heavy metals, DOS, absorption spectra, solvent polarity

Corresponding author: Ivan Shtepliuk,e-mail: ivan.shtepliuk@liu.se

\section{Introduction}

Among different toxic substances affecting the growing plants, human health and animals' lifecycle, a certain special role is played by heavy metals (HMs). The urgency of the problem 
of the environmental pollution by HMs stems from their extremely high toxicity and their uncontrollable release into the sea water, air and biosphere. By being involved into the biological cycle (“soil-plant-animal-human” and "water-animal-human” biosystems),they can have a significant long-term negative impact on human health. The three most hazardous and poisonous heavy metals are Cadmium (Cd), Mercury ( $\mathrm{Hg}$ ) and Lead (Pb).According to the recent official reports on the anthropogenic pollutants within European region, approximately 3467 tonnes of lead, 204 tonnes of cadmium and 167 tonnes of mercury are realized to the atmosphere annually. ${ }^{1}$ When exceeding the critical concentration limit, these hazardous substances can cause detrimental effects on the human health and environment. In particular, even small concentrations of divalent mercury ions $\left(\mathrm{Hg}^{2+}\right)$ are responsible for the dysfunction of the central nervous system, kidneys, liver, and brain. ${ }^{2}$ Divalent lead $\left(\mathrm{Pb}^{2+}\right)$ ions can damageirreversibly kidneys, brain, blood and muscles, thereby causing anemia, paralysis and mental-retardation. ${ }^{3}$ Cadmium $\left(\mathrm{Cd}^{2+}\right)$ is another highly toxic metal, which can lead to pathological problems with central nervous system, kidney's filtering system, and immune system. ${ }^{4}{ }^{5}$ For these reasons, it is vitally important to develop techniques for reliable monitoring of the heavy metals in the environment, drinking water, food, and biological liquids. Despite high sensitivity and acceptable selectivity, conventional bulky methods for heavy metal detection have several drawbacks, including time-consuming sample preparation procedure, strong demand in training professional staff, high cost of the detection tools. ${ }^{6-9}$ All these make the traditional heavy metals detectors non-real time, non-portable and highly expensive. Unlike bulky methods, sensors based of nanomaterials have attracted a great deal of attention because of the possibility touse them for on-site, selective and real-time detection of multiple heavy metals. Recent progress in the development of nanomaterials and their sensing applications accelerates the miniaturization of high performance sensors towardsimprovement of the sensitivity, detection limit, selectivity, and reproducibility.

Being a truetwo-dimensional crystal, grapheneis revolutionary carbon-based material, which possesses large surface area, chemical stability, exceptionally high electrical conductivity, a wide electrochemical potential window and high sensitivity to adsorbates or heteroatom doping. ${ }^{10,11}$ These features combined together create thedesired prerequisites of using graphene for electrochemical detection of the toxic heavy metals.To further improve the functionality of graphene in terms of sensing ability, the scientific community has extensively investigated a new class of low-dimensional graphene-based materials - 0D graphene quantum dots (GQDs). ${ }^{12,13}$ Owing to their tuneable emissive properties, graphene quantum dots can be employed for novel applications in bio-imaging ${ }^{14}$, optical sensing ${ }^{15}$ and detecting 
heavymetal ions in solutions. ${ }^{16,17}$ It is important to note that emission and/or absorption wavelength can be tuned by changing the size and edge termination ofGQDs. ${ }^{18,}{ }^{19}$ Putting the spotlight on GQDs as a promising nano-sized sensitive material, several groups reported successful identification of HMs by utilizing GQDs. ${ }^{20-22}$ Regarding electrochemical sensing, the functionalization of gold nanoparticles with GQDs was suggested as a promising route for improvement of the working electrode performance towards sensitive electrochemical detection of heavy metal ions..$^{20,21}$ Other authors reported on the fabrication of GQDs-encaged porous gold electrodes and GQDs-modificated glassy carbon electrodes for detection of $\mathrm{Pb}^{2+}$ ions. ${ }^{16,22}$ On the other hand, optical detection of $\left[\mathrm{Hg}^{2+}\right]^{23,24}$ and $\left[\mathrm{Pb}^{2+}\right]^{17,25}$ has been also demonstrated using GQDs. The GQD-mediated $\mathrm{Hg}^{2+}$ ions detection in pure aqueous solutions using fluorescence chemosensing have been reported. ${ }^{24}$ Alarge fluorescence quenching for GQDs was observed after complexation with divalent mercury ions.DNA aptamer-linked GQDs and GQD-DMA-tryptophan conjugates were utilized for $\mathrm{Pb}^{2+}$ detection by measuring the fluorescence quenching. ${ }^{17}$ It is believed that the fluorescence quenching mechanism is mainly governed by the electron transfer process from the GQDs to metal ions. ${ }^{25}$ Notwithstanding, this effect is only qualitatively explained and therefore deep understanding of the underlying physics behindthis quenching requires a theoretical support. In fact, two approaches to detect heavy metals using GQD have been reported: (i) modification of the working electrode surface with GQDs for the electrochemical detection of heavy metals and (ii) the use of GQDs for optical detection. In both cases, the target metal directly interacts with the GQDs, and therefore understanding the detection mechanism demands fundamental knowledge about the binding energy of the metal on graphene and about changing the electronic and optical properties of graphene under exposure to HMs.In our previous work, we investigated the adsorption of individual heavy metal atoms onto varying sized armchair- and zigzag-edged hydrogenated GQDs. ${ }^{19}$ The most energetically preferable adsorption sites for cadmium, mercury and lead were determined and the electronic and optical properties of GQDs before and after complexation with HMs were discussed.It should be noted that the results obtained and conclusions are valid for the case when the physisorption or chemisorption of metals on the surface of a graphene quantum dot occurs in a medium with relative permittivity very close to the vacuum value of unity and any factors that facilitate screening of the "GQD-HM" interaction are excluded or minimized. Nevertheless, in many cases,commercially available GQDs are dispersed in different organic solvents. ${ }^{11,26}$ Due to this reason, the interaction between heavy metals and graphene quantum dots can occur in liquid environment and thus specific interaction between the solvent and the 
solute substances must be considered.An important role of the solvent on the emission properties of nitrogen-doped GQDs has been shown earlier. ${ }^{27}$ In particular, it was found that the solvent changes from protic $\left(\mathrm{H}_{2} \mathrm{O}\right)$, aprotic (DMF) to solvent free causea shift of the emission of $\mathrm{N}$-doped GQDs from blue, green to yellow, respectively.The authors explained the observed shift of the emission peak by the influence of the solvent on the shape and composition of GQDs. An increase in the size of GQDs leads to a decrease in their energy gap and, as a consequence, a red shift in the emission peak.Zhaoet al.investigated theoretically the solvent effect on the absorption spectra of the GQDs consisting of 132 carbon atoms. ${ }^{28}$ In contrast to GQD in gas phase, a red shift (approximately $20 \mathrm{~nm}$ ) in the absorption wavelength of the GQD in solvent (toluene or dichloromethane) and a significant increase in its oscillator strength was observed.Niu et al. have found a similar effect in the case of the nitrogen doped GQD in the toluene and THF solvents. ${ }^{29}$ Taking the aforementioned into account, we anticipate that the solvents can affect the charge transfer process between GQD and HMs, thereby modifying the electronic and optical properties of the interacting systems.To our best knowledge, this matter has not been reported yet.Here we consider the solvent-mediated interaction between three most toxic heavy metals $(\mathrm{Cd}, \mathrm{Hg}$, and $\mathrm{Pb})$ and zigzag/armchair-edged graphene quantum dots. To investigate the solvent effect, we calculated the binding ability of GQDs to different heavy metals as well as electronic density of states and absorption spectra of interacting HMs@GQDs complexes in the gas phase, water, acetic acid and n,n-dimethylformamide (DMF).We are aiming to gain deep insights into the nature of the excited transitions in GQDs before and after complexation in different media.

\section{Theoretical Approach}

All quantum chemistry calculations reported in this paper were performed using the Gaussian 09 Rev. D.01 program package. ${ }^{30}$ The interaction of the neutral and divalent heavy metal species $\left(\mathrm{Cd}^{0}, \mathrm{Hg}^{0}, \mathrm{~Pb}^{0}\right.$ and $\left.\mathrm{Cd}^{2+}, \mathrm{Hg}^{2+}, \mathrm{Pb}^{2+}\right)$ with both zigzag (ZZ) and armchair (AC)GQDshaving similar number of carbon atoms was examined. Geometry optimization of the interacting systems in the gas-phase and different solvents (water, DMF, ethanol, and acetic acid) was done at the Becke three-parameter Lee-Yang-Parr hybrid (B3LYP) level of $\mathrm{DFT}^{31}$ with a 6-31G basis set for carbon and a basis set developed by the Stuttgart-DresdenBonn group for the heavy metal atoms ${ }^{32}$, using the default convergence criteria. In order to investigate the solvent effect on the interaction between HM species and GQDs as well as the properties of the GQDs before and after complexation with HMs the Polarizable Continuum Model (PCM) method in Gaussian 09 Rev. D.01 was applied. ${ }^{33}$ We consider $\mathrm{C}_{54} \mathrm{H}_{22}$ and 
$\mathrm{C}_{54} \mathrm{H}_{18}$ as representatives of armchair- and zigzag-edged GQDs, respectively (Figure 1). The cohesive energy per atom is defined as the energy required for separating the pristine GQD into isolated free atoms. We determined this energy as follows:

$E_{\text {coh }}=\frac{E_{G Q D}-\left(\sum_{i} E_{C}+\sum_{k} E_{H}\right)}{n_{C}+n_{H}}$

where $E_{G Q D}$ is the total energy of the relaxed armchair- or zigzag-edged GQD, $E_{C, H}$ the energy of isolated carbon and hydrogen atoms, respectively.The indices iand kimply that the summation goes over all carbon (C) and hydrogen (H) atoms in GQD, while $n_{C, H}$ defines the amount of the atoms in the considered systems.

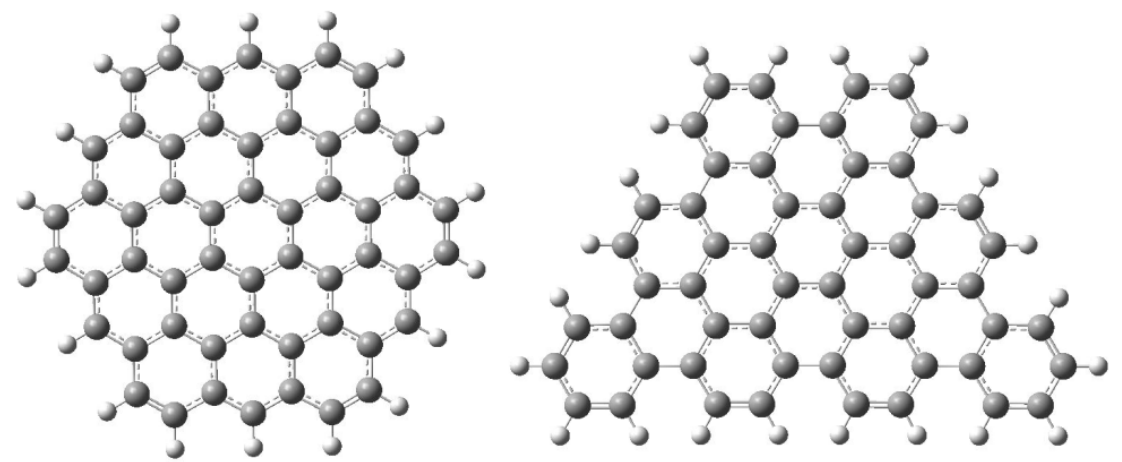

Figure 1. Optimized structures of the zigzag-edged $\mathrm{C}_{54} \mathrm{H}_{18} \mathrm{GQD}$ (left panel) and armchair-edged $\mathrm{C}_{54} \mathrm{H}_{22} \mathrm{GQD}$ (right panel). Small balls correspond to hydrogen atoms; large atoms represent carbon species belonging to GQD.

Future development of high-performance HMs detectors requires fine tuning of the reactivity of the GQDs with HM species. In line with this, solution phase chemistry engineering may be useful in controlling the binding ability of the zigzag- and armchair-edged GQDs to HMs exposure and, as a consequence, in improving the sensitivity and selectivity of the related devices. For this reason, the investigations of the interacting systems towards binding energy, charge transfer process, solubility and stability are highly desired to bring an understanding of the controllable solvent-mediated interaction between HMs and GQDs. Therefore, we started with calculation of the binding energy of the heavy metal species to a graphene quantum dot by the following equation:

$$
E_{b}=-\left[E_{G Q D+H M}-\left(E_{G Q D}+E_{H M}\right)\right]
$$

where $E_{G Q D+H M}$ is the total energy of the GQD after complexation with HM, $E_{G Q D}$ is the total energy of the isolated individual GQD and $E_{H M}$ is the total energy of an individual neutral atom 
or divalent ion of the HM. It should be mentioned that the binding energies have been corrected for the basis set superposition error (BSSE) by means of counterpoise method. ${ }^{34}$ Solubility of GQDs with and without HMs can be estimated as a difference between the optimized energies of the pristine/interacting systems in the solvent (PCM calculations) and vacuum (gas phase calculations) by the relation:

$$
\Delta E_{\text {solv }}=E_{\text {tot }-P C M}-E_{\text {tot }-G P}
$$

In all cases, the solubility of a GQDs in different liquids demandsa negativevalue of $\Delta E_{\text {solv }}$.To gain insight into the reactivity of GQDs we calculated the global reactivity descriptors ${ }^{35-37}$, such as global hardness, $\eta$, and electrophilicity, $\omega$, by using the relations below:

$$
\left\{\begin{array}{c}
\eta=\frac{E_{L U M O}-E_{\text {HOMO }}}{2} \\
\omega=\frac{\left(E_{L U M O}+E_{\text {HOMO }}\right)^{2}}{4\left(E_{\text {LUMO }}-E_{\text {HOMO }}\right)}
\end{array}\right.
$$

where $E_{\text {номо }}$ is the energy of the highest occupied molecular orbital (HOMO) and $E_{\text {LUмо }}$ is the energy of the lowest unoccupied molecular orbital (LUMO). The charge transfer is calculated using the Mulliken charge analysis.

The main part of the paper is dedicated to an analysis of the origin of the excited transitions in pristine GQD s and HM@GQDS. With this aim we calculated the ultravioletvisible (UV-vis) absorption spectra of the zigzag and armchair-edged GQDs by using the time-dependent density functional theory (TD-DFT) approach at the TDDFT/B3LYP level of theory using 6-31G basis set, implemented in the Gaussian 09 Rev. D.01 program. 100 excited electron states - transitions between occupied and unoccupied states - were involved to calculate the absorption spectra of the pristine GQDs and HM@GQDs interacting systems in the gas-phase and different solvents. It is worth nothing that the oscillator strengths for each vertical transitions in the GQDs investigated define the intensities of absorption peaks. We anticipate that the charge transfer process between GQDs and HM species will cause shift and quenching of the absorption peaks. Thus, a monitoring of the changes in optical properties of the GQDs is a helpful approach to estimate the binding strength between HMs and GQDs.

\section{Results and discussions}

This Section is organized as follows. Subsection 3.1 starts withdescribing of the theoretical results obtained to understand how solvent type influences the electronic and optical properties of the pristine zigzag- and armchair-edged GQDs. This subsection provides the scientific background for further discussions. Sections 3.2 describes the new insights into the 
physical nature of the optical and electronic response of the GQDs to exposure to neutral HM adatoms. Subsection 3.3 then combines the results on investigations of the binding ability of the GQDs immersed in different media to divalent HM ions. Subsection 3.4 includes general discussion on how obtained results can be usedfor understanding the mechanisms underlying the electrochemical detection of HM ions different liquids. Here we will shed light on the physics underlying the phenomena of excited transitions in GQDs after complexation with charged HM species.

\subsection{Pristine GQDs: solvent effect}

The stability of the GQDs in different media is an important aspect of GQDs in sensing applications. In principle, knowledge about the stability of the GQDs can help to prepare the GQD solution electrolyte, providing a desired sensitivity/selectivity to HM adsorbates. Here we compare the cohesive energies of zigzag- and armchair-edged GQDs immersed in different dielectric media. Calculated results are given in Table 1.For all dielectric media, ZZGQDsare more stable than AC-GQDs. In particular, cohesive energy of ZZ-GQDs is $\sim 0.26 \mathrm{eV} \cdot$ atom $^{-1}$ greater than AC-GQDs. This difference is due to the different regularity and symmetry of the GQDsboundaries. In addition, our model implies an enhanced hydrogenation of the edges in the case of the AC-GQDs and, as a consequence, a lower energy barrier to be overcome before bonding breaking occurs. The calculated cohesive energy is in good agreement with previously reported value of the cohesive energy of carbon in graphene, 7.4 $\mathrm{eV}$ per $\mathrm{C}$ atom. ${ }^{38}$ It should be mentioned that this energy refers to carbon atoms belonging to inner hexagonal rings, while the carbon species at the edgesare expected to be less stronglybound.As one can see in Table 1, an increase in the solvent dielectric constant shows small effect on the cohesive energy (decrease only by 50 meV), indicating alowering of the stability of the GQDs. It is also evidenced by the changes in the electrophilicity index. This parameter gives an important information about the effect of the dielectric medium on the GQD stability. Increase in this index with increasing the dielectric permittivity means that the GQDs immersed in more polar solvents are less stable and more reactive. ${ }^{39,40}$

Changes in the cohesive energy are accompanied with changes in the solubility of GQDs (See Table 1). The calculated solvation energies, presented in Table 1, are negative in all cases. For both zigzag-edged and armchair-edged GQDs, the solvation energy is increased with increasing the solvent dielectric constant, reaching the maximum value for water. Furthermore, the solubility of GQDs in organic solvents depends on the edge configuration.If we comparethe solvation energy of the ZZ-GQDs with that of the AC-GQDs, we see that the solubility is larger for the AC-GQDs.A partial desolvation of the GQDs in organic solvents 
leads to changes in their electronic properties. In particular, with increasing the solvent dielectric constant the HOMO level shifts towards more negative values for both types of GQDs. Since theHOMO energy is an indicator of the GQDability to donate electrons to appropriate acceptors, then more negative HOMO energies imply easier and faster charge transfer process. In other words, GQDs dispersed in solvents with high dielectric constant are more reactive (more electrons are available for reaction) than those in the gas-phase. In

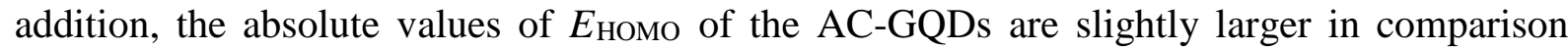
with those of ZZ-GQDs, suggesting abetter reactivity and binding ability to possible adsorbates in the case of armchair-type edge termination.Despite the slight drop of the HOMO level in a polar solvent, the HOMO-LUMO gap and global hardness is less sensitive to dielectric permittivity. These findings open the way for tuning the reactivity of GQDs by controlling the dielectric permittivity of a solvent.

Table 1. Computed parameters of zigzag- and armchair-edged GQDs immersed in different media

\begin{tabular}{|c|c|c|c|c|c|c|c|c|c|c|}
\hline \multirow{2}{*}{ Parameter } & \multicolumn{2}{|c|}{$\begin{array}{c}\text { Gas-phase } \\
\boldsymbol{\varepsilon}=\mathbf{1}\end{array}$} & \multicolumn{2}{c|}{$\begin{array}{c}\text { Acetic acid } \\
\boldsymbol{\varepsilon = 6 . 2 5 2 8}\end{array}$} & \multicolumn{2}{c|}{$\begin{array}{c}\text { Ethanol } \\
\boldsymbol{\varepsilon = 2 4 . 8 5 2}\end{array}$} & \multicolumn{2}{c|}{$\begin{array}{c}\text { DMF } \\
\boldsymbol{\varepsilon}=\mathbf{3 7 . 2 1 9}\end{array}$} & \multicolumn{2}{c|}{$\begin{array}{c}\text { Water } \\
\boldsymbol{\varepsilon}=\mathbf{7 8 . 3 5 5}\end{array}$} \\
\cline { 2 - 13 } & $\mathbf{Z Z}$ & $\mathbf{A C}$ & $\mathbf{Z Z}$ & $\mathbf{A C}$ & $\mathbf{Z Z}$ & $\mathbf{A C}$ & $\mathbf{Z Z}$ & $\mathbf{A C}$ & $\mathbf{Z Z}$ & $\boldsymbol{A C}$ \\
\hline $\mid$ Cohesive energy|,eV & 7.408 & 7.145 & 7.355 & 7.095 & 7.343 & 7.084 & 7.342 & 7.083 & 7.340 & 7.082 \\
\hline Solvation energy, eV & - & - & -0.356 & -0.411 & -0.464 & -0.536 & -0.478 & -0.552 & -0.493 & -0.570 \\
\hline HOMO, Hartree & -0.1886 & -0.1922 & -0.1954 & -0.1998 & -0.1978 & -0.2025 & -0.1981 & -0.2028 & -0.1984 & -0.2032 \\
\hline LUMO, Hartree & -0.0826 & -0.0751 & -0.0893 & -0.0827 & -0.0917 & -0.0854 & -0.0921 & -0.0857 & -0.0924 & -0.0861 \\
\hline $\boldsymbol{E}_{\text {Hомо-Lumo, } \mathbf{e V}}$ & 2.885 & 3.185 & 2.886 & 3.186 & 2.885 & 3.186 & 2.885 & 3.186 & 2.885 & 3.186 \\
\hline Electrophilicity, eV & 4.722 & 4.155 & 5.203 & 4.641 & 5.381 & 4.817 & 5.405 & 4.840 & 5.431 & 4.866 \\
\hline Hardness, eV & 1.442 & 1.592 & 1.443 & 1.593 & 1.442 & 1.593 & 1.442 & 1.593 & 1.442 & 1.593 \\
\hline
\end{tabular}

The optical properties of the GQDs immersed in different media here are best represented by the absorption spectra.The absorption spectra of zigzag- and armchair-edged GQDs are presented in Figure 2. Each spectrum exhibits one intense absorption band at $415 \mathrm{~nm}$ for ZZGQDs and $370 \mathrm{~nm}$ for AC-GQDs, respectively. As can be seen from Figure 2,the absorption wavelengthof both ZZ-GQDs and AC-GQDs dispersed in solvents is red shifted as compared to thosein the gas-phase (from $415 \mathrm{~nm}$ to $427 \mathrm{~nm}$ for ZZ-GQDs and from $370 \mathrm{~nm}$ to $378 \mathrm{~nm}$ for AC-GQDs, respectively). Furthermore, in both cases the absorption peak slightly increases in the intensity when passing from the gas-phase to solvents.The observed changes can be ascribed to the conductor-like screening effect, which is responsible for the appearance of the solvent-induced dipole moments and, as a consequence, for the modification of the configurations of the oscillators. ${ }^{41-43}$ Nevertheless, the absorption wavelengths and absorption intensities are almost similar in all considered solvents (acetic acid, ethanol, DMF and water). In fact, even the water, having the strongest polarity, has no significant effect on the optical 
properties of the graphene quantum dots in comparison to other solvents. To understand the origin of theabsorption peaks we performed DOS calculations for both GQDs in different media. It wasrevealed that the HOMO-1 and HOMO as well as LUMO+1 and LUMO are degenerate $\pi$ and $\pi^{*}$ orbitals in zigzag-edged GQDs (Figs. 3a and 3b, respectively). This is because they have the same energies, orbital compositions, and occupancy. Figs. 3a and 3b also illustrate the transitions from the occupied states to the empty states (since all solvents demonstrate similar effect on the absorption spectra of the ZZ-GQDs, here we compare only results of DFT calculations in the gas-phase and water). Thus, the observed absorption spectra of the ZZ-GQDs are originating from multilevel lowest singlet transitions between degenerated orbitals. In other words, the corresponding spectra are dominated by four different transitions:HOMO $\rightarrow$ LUMO (46\%) and H-1 $\rightarrow$ L+1 (46\%); H-1 $\rightarrow$ LUMO (46\%) and $\mathrm{HOMO} \rightarrow \mathrm{L}+1$ (46\%). Molecular orbitals, which are involved in these transitions are shown in Figure S1 (Electronic Supplementary Information \#2).
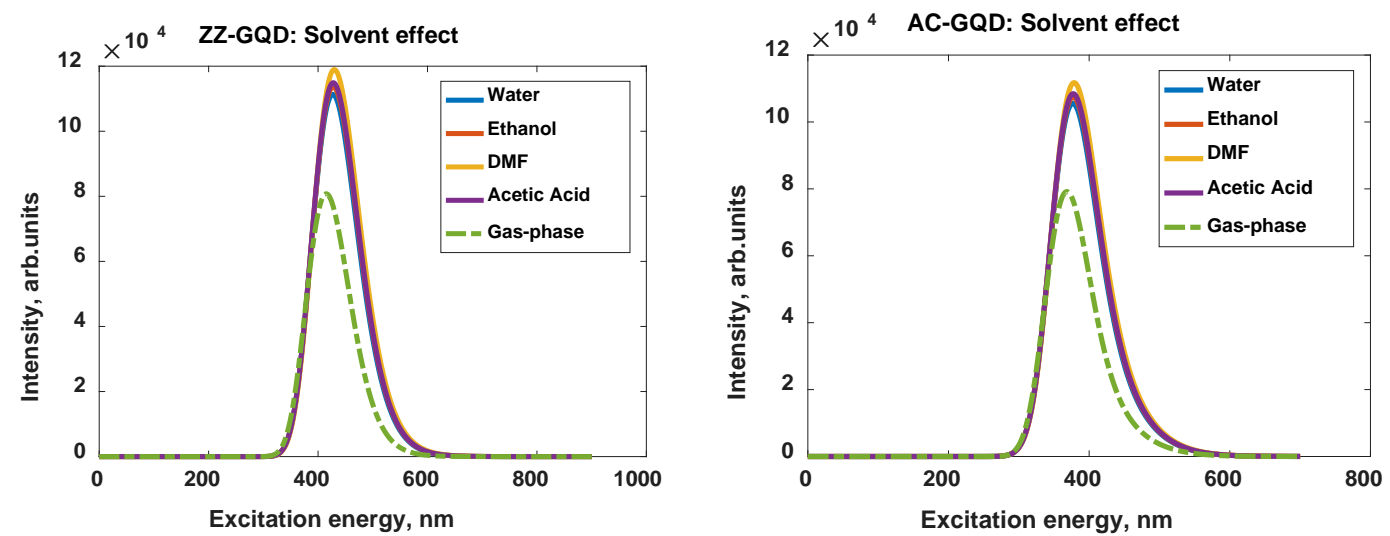

Figure 2. UV-vis absorption spectra of the pristine GQDs obtained in different dielectric media by using the PCM/TD-DFT/B3LYP/6-31G calculations of excited transitions: (left panel) zigzag edge termination and (right panel) armchair edge termination.

Contrary to the doubly degenerate LUMO and HOMO in ZZ-GQDs, the lowest orbitals in AC-GQDs split into two nondegenerate orbitals (see Figs. 3c and 3d, see also Figure S1, Electronic Supplementary Information\#2). The splitting of the LUMO and HOMO is responsible for a change in the nature of the electronic excitations: from doubly degenerate $\mathrm{HOMO} \rightarrow$ LUMO transition to two nondegenerate transitions $(369 \mathrm{~nm}$ and $371 \mathrm{~nm}$ in gasphase and 377 and $378 \mathrm{~nm}$ in water). Therefore, the absorption spectra of AC-GQDs are composed of two sets of transitions with similar oscillator strengths: $H-1 \rightarrow L+1$ (75\%), $\mathrm{HOMO} \rightarrow$ LUMO (16\%) as well as $\mathrm{H}-1 \rightarrow$ LUMO (46\%), HOMO $\rightarrow \mathrm{L}+1$ (48\%). We find also a weak contribution of electronic excitation at $350 \mathrm{~nm}$ (355 nm in water) with an oscillator 
strength of $f=0.326$ ( $f=0.319$ in water)to absorption spectra of the AC-GQDs. From the analysis of the electronic nature of this excitation, one can conclude that the dominant contribution to this transition arises from $\mathrm{H}-2 \rightarrow \mathrm{LUMO}$ and $\mathrm{HOMO} \rightarrow \mathrm{L}+2$.Tables $\mathrm{S} 1$ and S2 (Electronic Supplementary Information\#2) summarize the electronic transitions, which contribute to the absorption spectra of the ZZ-GQDs and AC-GQDs immersed in different media.
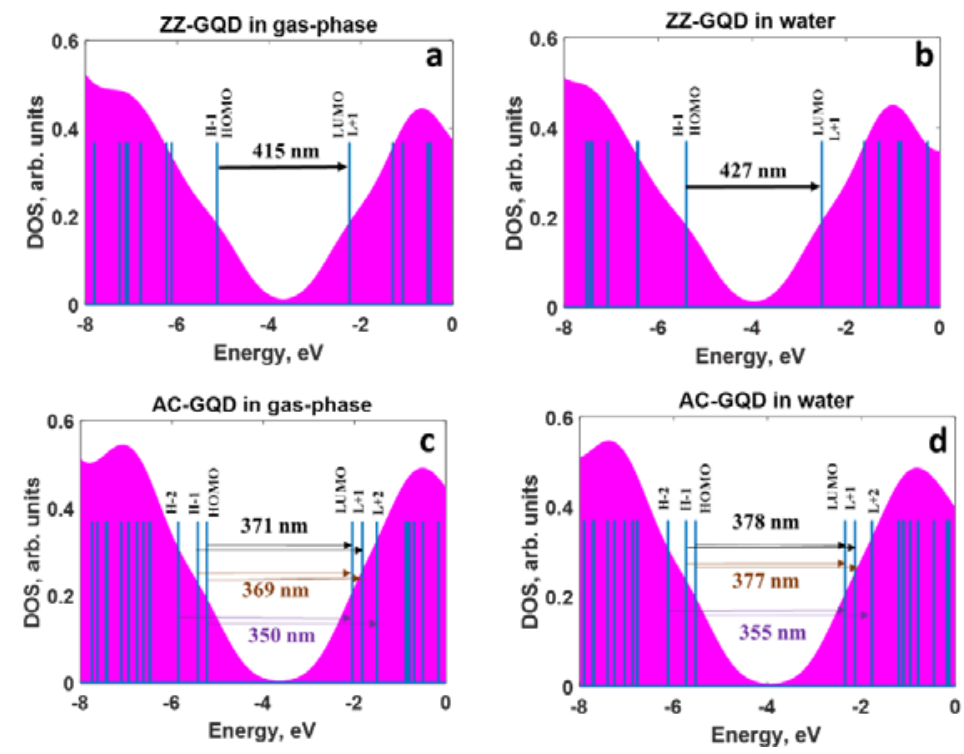

Figure 3. DOS of pristine GQDs (shaded area) immersed in different media: (a) ZZ-GQD in the gas-phase, (b)ZZ-GQD in the water, (c) AC-GQD in the gas-phase, (d)AC-GQD in the water. Blue vertical lines denote the molecular orbitals. The excited transitions between available occupied and unoccupied electronic levels are shown by black arrowed lines.

\subsection{Solvent-mediated interaction between GQDs and neutral adatoms of HMs:} geometry, electronic and optical properties.

\subsubsection{Cd $\mathbf{d}^{0} @ G Q D$}

It has been shown beforethat theadsorption of the neutral Cd atom is the most stable at the hollow site on both GQDs and extended graphene. ${ }^{19}$ The most favourable adsorption configuration in the presence of solvents, (see Table S3,Electronic Supplementary Information\#2), is similar to that in the gas-phase. Focusing on the stability of the GQDs after complexation with $\mathrm{Cd}^{0}$, we noticed that the global hardness gradually decreases with increasing of the dielectric constant of the solvent, reaching the minimal value for water, Table 2. The solvation energy becomes more negative as the solvent becomes more polar. In line with this, the electrophilicity index of the interacting system is also increased in the solvated phases. Such trends imply that the solvents diminish the stability of both $\mathrm{Cd}^{0} @ \mathrm{ZZ}-$ GQDs and $\mathrm{Cd}^{0} @ \mathrm{AC}-\mathrm{GQDs}$, thereby raising its reactivity. There is also a 
distinctincrease in the binding energy of neutral cadmium on ZZ-GQD (AC-GQD) ranging from $0.171(0.179) \mathrm{eV}$ for the gas-phase to $0.275(0.273) \mathrm{eV}$ for water, indicating a solventmediated enhancement of the interaction strength. For both edge configurations, the binding energy follows the order of $E_{b}$ in water $>E_{b}$ in $D M F>E_{b}$ in ethanol $>E_{b}$ in acetic acid $>E_{b}$ in gas phase. Interestingly, in the presence of solvent HOMO level of the ZZ-GQD and AC-GQD is upshifted by $0.2 \mathrm{eV}$ compared to the value in the gas-phase. Furthermore, the presence of solvents modulates the HOMO-LUMO gap energy, decreasing its value with increasing the solvent dielectric constant for Cd0@ZZ-GQDs and Cd0@@AC-GQDs.

Table 2. Computed parameters of zigzag- and armchair-edged GQDs after complexation with $C d^{0}$ in different media

\begin{tabular}{|c|c|c|c|c|c|c|c|c|c|c|}
\hline \multirow[t]{2}{*}{ Parameter } & \multicolumn{2}{|c|}{$\begin{array}{c}\text { Gas-phase } \\
\varepsilon=1\end{array}$} & \multicolumn{2}{|c|}{$\begin{array}{c}\text { Acetic acid } \\
\varepsilon=6.2528\end{array}$} & \multicolumn{2}{|c|}{$\begin{array}{c}\text { Ethanol } \\
\varepsilon=24.852\end{array}$} & \multicolumn{2}{|c|}{$\begin{array}{c}\text { DMF } \\
\varepsilon=37.219\end{array}$} & \multicolumn{2}{|c|}{$\begin{array}{c}\text { Water } \\
\varepsilon=78.355\end{array}$} \\
\hline & $C d^{0} @ Z Z$ & $C d^{0} @ A C$ & $C d^{0} @ Z Z$ & $C d^{0} @ A C$ & $C d^{0} @ Z Z$ & $C d^{0} @ A C$ & $C d^{0} @ Z Z$ & $C d^{0} @ A C$ & $C d^{0} @ Z Z$ & $C d^{0} @ A C$ \\
\hline Binding energy, eV & 0.171 & 0.179 & 0.267 & 0.251 & 0.284 & 0.269 & 0.269 & 0.271 & 0.275 & 0.273 \\
\hline Charge on Cd & 0.08 & 0.07 & 0.06 & 0.05 & 0.06 & 0.05 & 0.06 & 0.05 & 0.06 & 0.05 \\
\hline Solvation energy, eV & - & - & -0.637 & -0.669 & -0.791 & 0.840 & -0.793 & -0.861 & -0.818 & -0.885 \\
\hline HOMO, Hartree & -0.1903 & -0.1943 & -0.1783 & -0.1779 & -0.1747 & -0.1745 & -0.1743 & -0.1742 & -0.1738 & -0.1738 \\
\hline LUMO, Hartree & -0.0841 & -0.0772 & -0.0895 & -0.0829 & -0.0916 & -0.0852 & -0.0918 & -0.0855 & -0.0921 & -0.0858 \\
\hline$E_{\text {номо-LUмо }}, \mathrm{eV}$ & 2.889 & 3.186 & 2.417 & 2.585 & 2.263 & 2.431 & 2.245 & 2.413 & 2.225 & 2.393 \\
\hline Electrophilicity, eV & 4.829 & 4.282 & 5.494 & 4.870 & 5.804 & 5.138 & 5.838 & 5.175 & 5.885 & 5.215 \\
\hline Hardness, eV & 1.444 & 1.593 & 1.208 & 1.292 & 1.131 & 1.215 & 1.122 & 1.206 & 1.112 & 1.196 \\
\hline
\end{tabular}

To further elucidate the nature of the solvent effect on the properties of the zigzag- and armchair-edged GQDs conjugated with $\mathrm{Cd}^{0}$, we investigate the absorption spectra of the aforementioned interacting systems in different media. In Fig. 4, we can find that both Cd $@$ @Z-GQDs and Cd ${ }^{0} @ A C-G Q D s$ in the gas-phase have a strong absorptions peaks at 415 $\mathrm{nm}$ and $370 \mathrm{~nm}$, respectively.Due to the physisorption nature of theinteraction between Cd and GQDs, these spectra are almost similar to those of pristine GQDs.
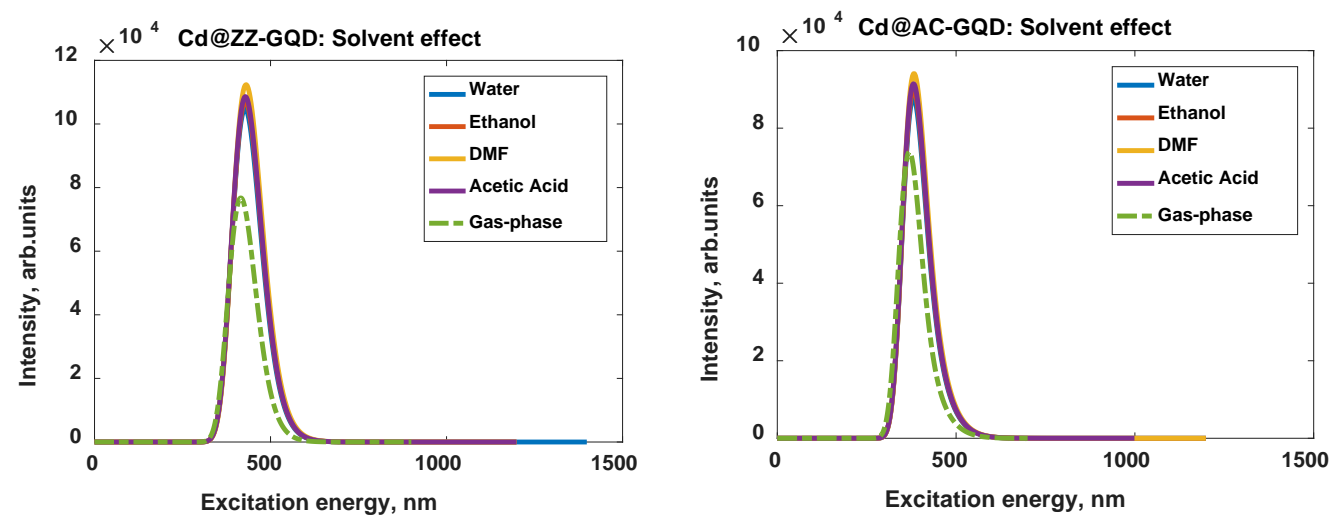

Figure 4. UV-vis absorption spectra of the GQDs after complexation with $C^{0}$ obtained in different dielectric media by using the PCM/TD-DFT/B3LYP/6-31G calculations of excited transitions: (left panel) zigzag edge termination and (right panel) armchair edge termination. 
To reveal the origin of the electronic transitions, molecular orbital composition analysis was performed. It was found that the Cd atom does not contribute to the HOMO, HOMO-1 andLUMO, L+1, while the HOMO-2 is entirely localized on Cd atom (see Figure S2, Electronic Supplementary Information\#2). To demonstrate detailed transitions, DOS of the interacting system with corresponding molecular orbital structure are presented in Fig. 5a. It is clearly seen that doubly degenerate excited state defines completely the absorption spectra of the zigzag-edged graphene quantum dots after complexation with neutral Cd atom (see DatasetS1, Electronic Supplementary Information\#2), and the Cd-related molecular orbitals are not involved in electronic transitions. When thearmchair-edged GQDs interact with Cd (in the gas-phase), more complex molecular orbital structure is observed and hybrid orbitals aremostly participating in the lowest-energy excitation. Furthermore, unlike zigzag-edged GQDs, where the prevailing doubly degenerate levels owe their origin to the symmetry, in the case of the armchair-edged GQD these levels disappear completely or partially as the symmetry breaks. Two electronic transitions with largest oscillator strengths involve HOMO1 and HOMO-2 levels (Fig. 5c), which are shared by cadmium adsorbate and graphene plane (see Figure S2 and DatasetS1, Electronic Supplementary Information\#2).While other orbitals involved in the excited transitions are delocalized over the planar structure of the AC-GQD.
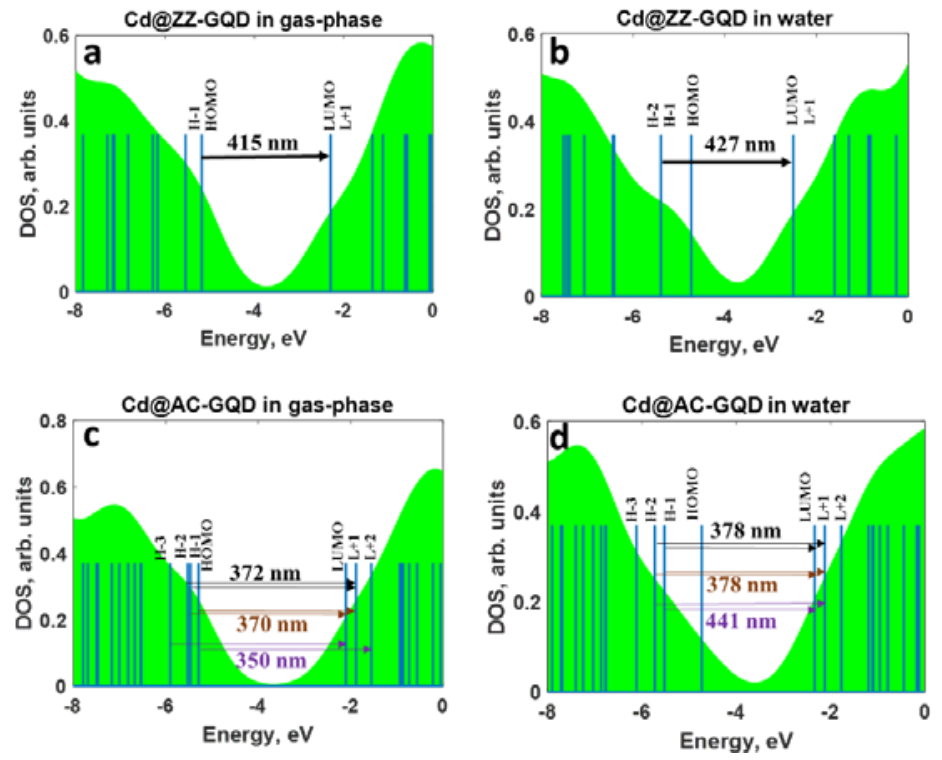

Figure 5. DOS of GQDs after complexation with $C d^{0}$ (shaded area) immersed in different

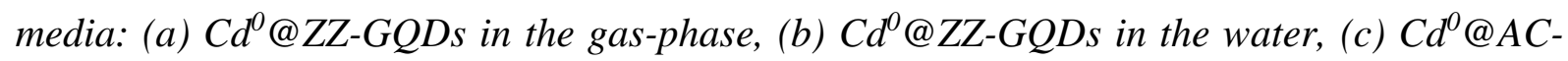
GQDs in the gas-phase, (d) Cd $@ A C-G Q D s$ in the water. Blue vertical lines denote the molecular orbitals. The excited transitions between available occupied and unoccupied electronic levels are shown by black arrowed lines. Note that, for simplicity, here we compare 
only two cases of the complexation of GQDs with Cd: in the gas-phase and water. Other solvents exhibit similar effect to water.

Both Cd ${ }^{0} @ Z Z-G Q D s$ and Cd ${ }^{0} @ A C-G Q D s$ undergo an obvious red-shift of their absorption curves when the dielectric constant of the solvent is increased (Fig. 4). This red-shift is in line with solvent-induced HOMO-LUMO band gap shrinking, which is related to a lowering of the LUMO level and a contemporary rise of the HOMO.It is worth mentioning that the absorption intensities of the interacting systems in the solvated phases are higher than that in vacuum, but do not changethe own value from solvent to solvent. These results were confirmed by the analysis of the oscillator strengths for most probable excited transitions, which in addition put into evidence an electronic transition at longer wavelength, independent of the dielectric constant of the solvent (see Dataset S1, Electronic Supplementary Information\#2). As was mentioned before, the interaction strength between GQDs and Cd adatom is enhanced with increasing the solvent polarity. In the case of water as a solvent - the most polar solvent among others - this effect manifests itself in the change of the molecular orbital structure and nature of the excited transitions (Figure 5, and Table S4). In particular, HOMO inCd ${ }^{0} @ Z Z-G Q D s$ is entirely localized on the Cd atom (Figure S2, Electronic Supplementary Information \#2). However, this orbital is not involved in electronic transitions. In fact, only electronic transitions between doubly degenerate occupied states(HOMO/HOMO-1) and empty levels (LUMO/LUMO+1) contribute tothe absorption spectra of Cd ${ }^{0} @ Z Z-G Q D s$.

Let us next look into the electronic transitions in the $\mathrm{Cd}^{0} @ \mathrm{AC}-\mathrm{GQDs}$ immersed in water solution. We can see from Fig. 5d that the absorption features observed near $378 \mathrm{~nm}$ correspond to the mixed transitions from the HOMO-1 and HOMO-2 to LUMO and LUMO+1.The other feature at $\sim 441 \mathrm{~nm}$ with oscillator strength of 0.2511 is associated with the following transitions: HOMO-2 $\rightarrow$ LUMO+1 (13\%), HOMO-1 $\rightarrow$ LUMO (86\%). It should be noted that all mentioned molecular orbitals are energetically stabilized over GQDs, while Cd-induced HOMO level does not contribute significantly to excited transitions. The first mainabsorption feature at 378 nmappearsrathersimilarto that of $\mathrm{Cd}^{0} @ A C-G Q D s$ in the gas phase, as observed in the Figs. 5c and 5d. However, the absorption curves in the latter two cases have entirely different origins. In the gas-phase,the electronic transitions are associated with the HOMO-1 and HOMO-2 hybridized orbitals, while DOS calculations in water suggest that wave functions of the HOMO-1 and HOMO-2 arehighly delocalized over ACGQD (Figure S2, Electronic Supplementary Information \#2). This finding means that there is 
a possibility to modulate the absorption intensity of Cd $\mathrm{d}^{0} @ \mathrm{AC}-\mathrm{GQD}$ at desired wavelength by deactivating hybridized complex states via control of the dielectric permittivity of the medium.

\subsubsection{Hg0@GQD}

Here wediscuss solvent effect on the binding ability of graphene quantum dots with different edge termination towards atomicmercury $\left(\mathrm{Hg}^{0}\right)$ and compare the calculated results for the gasphase with those for different solvents. Independently of the dielectric permittivity value for both ZZ-GQDs and AC-GQDs, the energetically most favourable Hg adsorption site is the hollow site (See Table S4, Electronic Supplementary Information\#2), which is in good agreement with previous studies of the interaction between neutralHg with GQD and extended graphene. ${ }^{19}$ The binding energy of $\mathrm{Hg}$ on the planar surface of ZZ-GQDs and ACGQDs as a function of dielectric constant of the solvent is listed in Table 3.

Table 3. Computed parameters of zigzag-and armchair-edged GQDs after complexation with $\mathrm{Hg}^{0}$ in different media

\begin{tabular}{|c|c|c|c|c|c|c|c|c|c|c|}
\hline \multirow[t]{2}{*}{ Parameter } & \multicolumn{2}{|c|}{$\begin{array}{c}\text { Gas-phase } \\
\varepsilon=1 \\
\end{array}$} & \multicolumn{2}{|c|}{$\begin{array}{c}\text { Acetic acid } \\
\varepsilon=6.2528 \\
\end{array}$} & \multicolumn{2}{|c|}{$\begin{array}{c}\text { Ethanol } \\
\varepsilon=\mathbf{2 4 . 8 5 2} \\
\end{array}$} & \multicolumn{2}{|c|}{$\begin{array}{c}\text { DMF } \\
\varepsilon=37.219 \\
\end{array}$} & \multicolumn{2}{|c|}{$\begin{array}{c}\text { Water } \\
\varepsilon=78.355 \\
\end{array}$} \\
\hline & $\mathrm{Hg}^{0} @ Z Z$ & $H g^{0} @ A C$ & $\mathbf{H g}^{0} @ Z Z$ & $H g^{0} @ A C$ & $H g^{0} @ Z Z$ & $\mathrm{Hg}^{0} @ A C$ & $H g^{0} @ Z Z$ & $H g^{0} @ A C$ & $\mathrm{Hg}^{0} @ Z Z$ & $\mathrm{Hg}^{0} @ A C$ \\
\hline Binding energy, eV & 0.162 & 0.164 & 0.309 & 0.307 & 0.356 & 0.335 & 0.342 & 0.339 & 0.358 & 0.342 \\
\hline Charge on $\mathrm{Hg}$ & 0.095 & 0.091 & 0.076 & 0.069 & 0.078 & 0.069 & 0.081 & 0.069 & 0.079 & 0.069 \\
\hline Solvation energy, eV & - & - & -0.755 & -0.805 & -0.949 & -0.998 & -0.954 & -1.022 & -0.989 & -1.048 \\
\hline HOMO, Hartree & -0.1899 & -0.19378 & -0.1953 & -0.19980 & -0.1975 & -0.20216 & -0.1977 & -0.20247 & -0.1981 & -0.20282 \\
\hline LUMO, Hartree & -0.0837 & -0.07670 & -0.0892 & -0.08273 & -0.0914 & -0.08509 & -0.0916 & -0.08540 & -0.0920 & -0.08574 \\
\hline$E_{\text {номо-Luмо, }}, \mathrm{eV}$ & 2.889 & 3.185 & 2.887 & 3.185 & 2.886 & 3.185 & 2.887 & 3.185 & 2.886 & 3.185 \\
\hline Electrophilicity, eV & 4.799 & 4.250 & 5.190 & 4.638 & 5.356 & 4.794 & 5.369 & 4.815 & 5.397 & 4.838 \\
\hline Hardness, eV & 1.444 & 1.59 & 1.443 & 1.592 & 1.443 & 1.592 & 1.443 & 1.592 & 1.443 & 1.592 \\
\hline
\end{tabular}

The calculated data suggest that Hgadatom binds strongly with GQDsin the presence of solvents. In particular, it is obvious that the binding energy increasedsignificantly for solvents with high polarity (almost 2 times enhancement), which indicates that anenhancedattractive interaction occurs between GQDs and $\mathrm{Hg}^{0}$. Such phenomena can be explained in terms of partial desolvation of both, Hg and GQDs. In this context, the reorganisation of solvent-Hg and solvent-GQD interactions upon complexation is responsible for the changes in the binding energy. In contrast to ZZ-GQD possessing zero dipole moment, the armchair-edged GQD has larger dipole moment and thus its solvation energy is higher. After complexation with $\mathrm{Hg}$ the dipole moment and solvation energy are increased because solventsare believed to optimize the adsorption geometry byminimizing the distance between Hg adsorbateand planar surface of the GQDs.The electrophilicity index of the Hg@ZZ-GQDs and Hg@ACGQDs follows clearly the solvation trend: the higher the desolvation of the interacting systems the higher the value of the electrophilicity index. It is worth mentioning that the 
HOMO-LUMO energy gap and the global hardness for Hg@ZZ-GQDs and Hg@AC-GQDs remain virtually unchanged with increase in dielectric permittivity implying relatively high stability of the interacting systems and proportionally equivalent changes in the HOMO/LUMO levels.

Finally, our results show that the solvent has a little influence on absorption spectra of the Hg@ZZ-GQDs and Hg@AC-GQDs (Fig. 6). This is similar to the case of the absorption spectra of the $\mathrm{Cd}^{0} @$ GQDs dispersed in different media, as has been shown in previous section. In the gas-phase, the absorption peaks of Hg@ZZ-GQDs and Hg@AC-GQDs appear at the visible bands of $415 \mathrm{~nm}$ as well as $350 \mathrm{~nm}$ and $370 \mathrm{~nm}$, respectively. These spectra display a small red shift (approximately $8 \mathrm{~nm}$ ) and slight increase in intensity when passing from the gas-phase to solution (Figure 6 and Figure7). In water, the mainabsorption peak of Hg@ZZ-GQDs is located at 427 nm, while the absorption features of the Hg@AC-GQDs appear at the visible bands of $355 \mathrm{~nm}$ and $378 \mathrm{~nm}$.
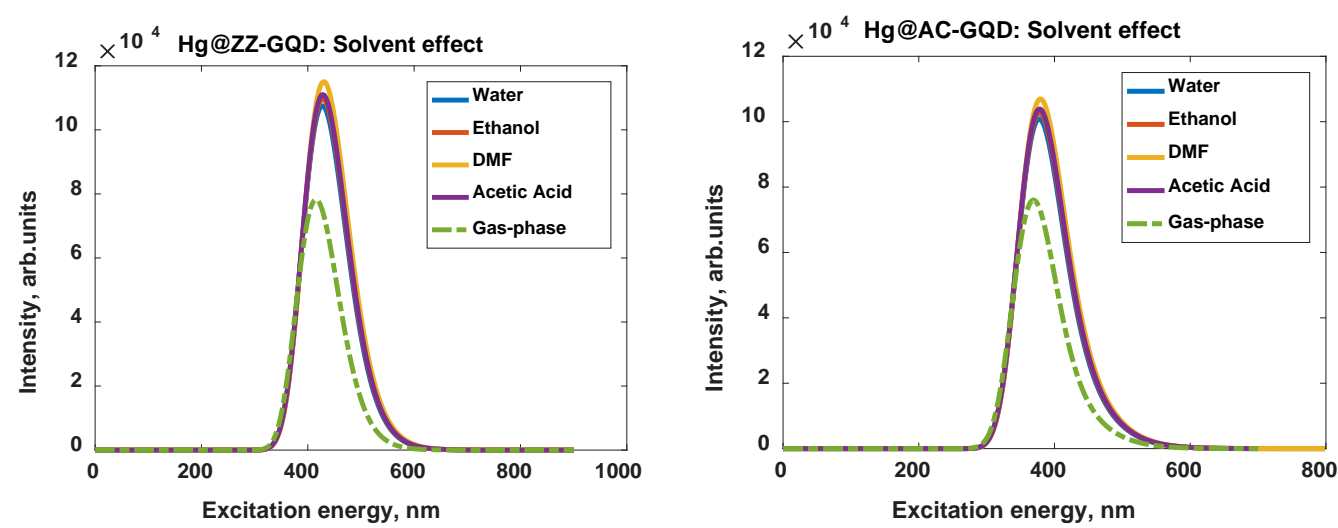

Figure 6. UV-vis absorption spectra of the GQDs after complexation with $\mathrm{Hg}^{0}$ obtained in different dielectric media by using the PCM/TD-DFT/B3LYP/6-31G calculations of excited transitions: (left panel) zigzag edge termination and (right panel) armchair edge termination.

Let us next shed some light on the nature of the excited electronic transitions underlying the bands in the absorption spectra and the role of $\mathrm{Hg}$ adatom in these transitions.For the Hg@ZZ-GQD in the gas-phase, it is found that the most intensive bandis a result of the configuration interaction between the HOMO $\rightarrow$ LUMO, HOMO-1 $\rightarrow$ LUMO +1 , HOMO$1 \rightarrow$ LUMOand $\mathrm{HOMO} \rightarrow$ LUMO +1 transitions. Since these transitions have identical energies, the excited state is accidentally degenerate.At the same time, the absorption features are mainly arising from the molecular orbitals, which are delocalized over the surface of the ZZGQDs. Due to the weak physisorption of Hg on ZZ-GQD, the first occupied electronic state, which is entirely localized on the Hg adatom is only HOMO-5. It is obvious that this Hg- 
related level does not participate in the excited transitions. On the other hand, due to the solvent polarity effect the interaction between ZZ-GQDs with atomicmercury is enhanced and, as a consequence,the wave function of HOMO-2 is highly localized on Hg. However, even in solvent with strong polarity the excited state is still mostly composed of mixture of double degenerate transitions with higher oscillator strengths and the corresponding molecular orbitals are completely delocalized over the ZZ-GQD.

For Hg@AC-GQD in the gas phase, there are two strong transitions between nondegenerate states. As can be seen in Fig. 7c, the transitions HOMO $\rightarrow$ LUMO, HOMO- $\rightarrow$ LUMO +1 , HOMO- $1 \rightarrow$ LUMO, HOMO $\rightarrow$ LUMO+1 and, to a less extent,HOMO-2 $\rightarrow$ LUMO contribute strongly to the absorption at $\sim 370-372 \mathrm{~nm}$. While the state at $350 \mathrm{~nm}$ with small oscillator strength is dominated by HOMO $-2 \rightarrow$ LUMO and $\mathrm{HOMO} \rightarrow \mathrm{LUMO}+2$ transitions. In the solvated phase (for example, water), the low-energy part of the absorption spectra contains a group of at least three electronic transitions corresponding to the HOMO $\rightarrow$ LUMO, HOMO$2 \rightarrow \mathrm{LUMO}+1, \quad \mathrm{HOMO}-2 \rightarrow \mathrm{LUMO}, \quad \mathrm{HOMO} \rightarrow \mathrm{LUMO}+1, \quad \mathrm{HOMO}-3 \rightarrow$ LUMO and $\mathrm{HOMO} \rightarrow \mathrm{LUMO}+2$ (Fig. 7d). In fact, electron density distribution for lowest energy molecular orbitals involved in excited transitions for Hg@AC-GQD in different media suggests a negligible role of the Hg-related states (Dataset 2, Electronic Supplementary Information\#2).
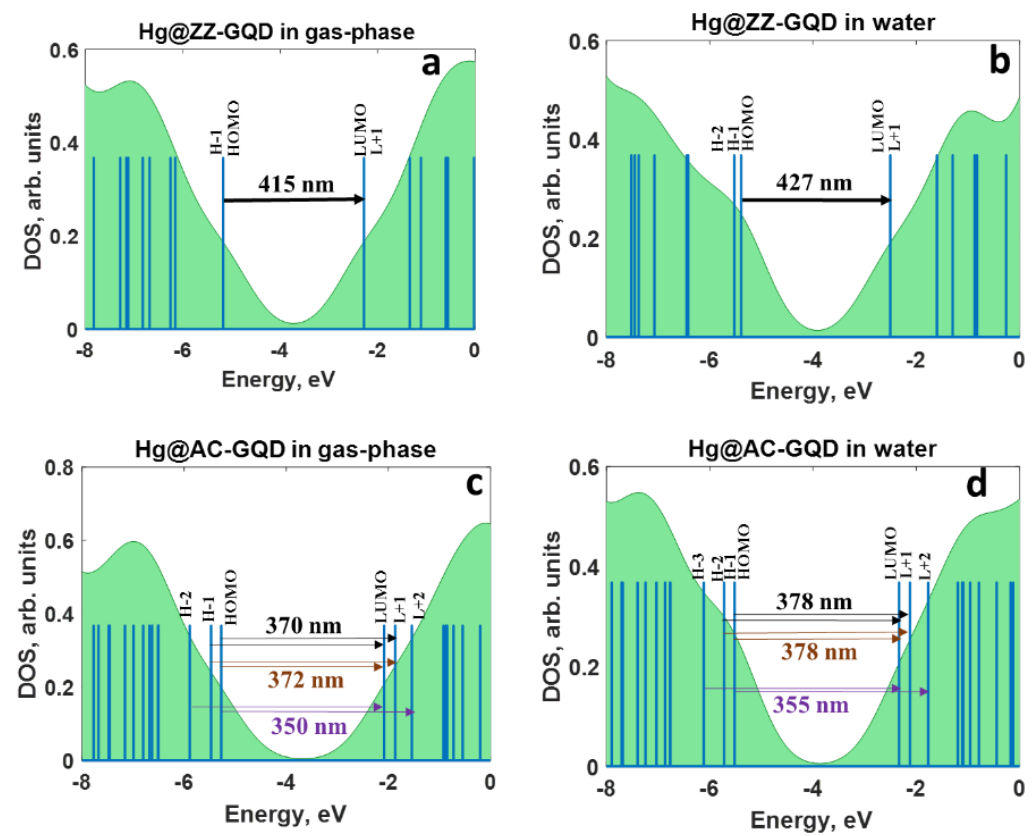

Figure 7. DOS of GQDs after complexation with $\mathrm{Hg}^{0}$ (shaded area) immersed in different

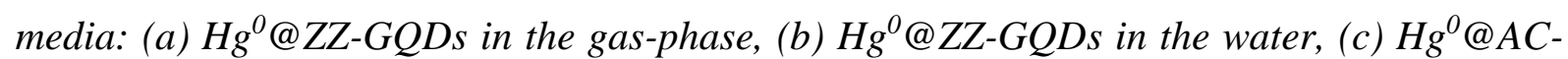
GQDs in the gas-phase, (d) $\mathrm{Hg}^{0} @ A C-G Q D s$ in the water. Blue vertical lines denote the 
molecular orbitals. The excited transitions between available occupied and unoccupied electronic levels are shown by black arrowed lines.

\subsubsection{PB' $\mathbf{P b}^{0} @ G Q D$}

A more complex bonding picture is observed in the case of interaction between GQDs and neutral $\mathrm{Pb}$ adatom. First of all, similarly to other heavy metals ( $\mathrm{Cd}$ and $\mathrm{Hg}$ ) in the gas-phase, $\mathrm{Pb}$ is electron-donating adsorbant, which is able to transfer electrons to the GQD easier than $\mathrm{Cd}^{0}$ and $\mathrm{Hg}^{0}$ adatoms (Table 4).This difference can be understood in terms of ionization potential. ${ }^{44}$ The ionization potential of $\mathrm{Pb}^{0}$ is approximately equal to $7.41 \mathrm{eV}$, while $\mathrm{Cd}$ and $\mathrm{Hg}$ have larger ionization energies of $8.99 \mathrm{eV}$ and $10.43 \mathrm{eV}$, respectively. ${ }^{45,46}$ The higher the ionization energy, the more difficult is to remove an electron from a metal adatom. Due to this reason the enhanced charge transfer from $\mathrm{Pb}$ to both ZZ-GQD and AC-GQD occurs. Secondly, our results indicate that $\mathrm{Cd}$ and $\mathrm{Hg}$ adatoms are always physisorped on the hollow sitesofthe surface of the GQDs with different edge termination. On the contrary, Pb adatom prefers to sit on bridge sites when adsorped. The bonding peculiarities for $\mathrm{Pb}$ on ZZ-GQD and AC-GQD in different media are illustrated by the optimized adsorption configurations (see Table S5, Electronic Supplementary Information\#2). The Pb adatom has approximately the same local position in all cases- bridge site, independently of the dielectric constant of the solvent. Another important observation is that an increase in dielectric constant leads to a change in direction of charge-transfer. In particular, when passing from the gas-phase to acetic acid the charge-transfer is initially decreased to approximately zero value and then changes the sign for solvents with high dielectric permittivity (Table 4). Thus, solvents with high polarity change the electroactive behaviour of the $\mathrm{Pb}$ adatom on graphene quantum dots from electron-donating to electron-accepting. That is, such dielectric media weaken the interactions between GQD surfaces and $\mathrm{Pb}$ adsorbate, as it was confirmed by a dramatic decrease in the binding energy with the increase of the dielectric constant of the solvent.

Table 4. Computed parameters of zigzag- and armchair-edged GQDs after complexation with $\mathrm{Pb}^{0}$ in different media

\begin{tabular}{|c|c|c|c|c|c|c|c|c|c|c|}
\hline \multirow[t]{2}{*}{ Parameter } & \multicolumn{2}{|c|}{$\begin{array}{c}\text { Gas-phase } \\
\varepsilon=1\end{array}$} & \multicolumn{2}{|c|}{$\begin{array}{c}\text { Acetic acid } \\
\varepsilon=6.2528\end{array}$} & \multicolumn{2}{|c|}{$\begin{array}{c}\text { Ethanol } \\
\varepsilon=24.852 \\
\end{array}$} & \multicolumn{2}{|c|}{$\begin{array}{c}\text { DMF } \\
\varepsilon=37.219\end{array}$} & \multicolumn{2}{|c|}{$\begin{array}{c}\text { Water } \\
\varepsilon=78.355\end{array}$} \\
\hline & $P b^{0} @ Z Z$ & $P b^{0} @ A C$ & $P b^{0} @ Z Z$ & $P b^{0} @ A C$ & $P b^{0} @ Z Z$ & $P b^{0} @ A C$ & $P b^{0} @ Z Z$ & $P b^{0} @ A C$ & $P b^{0} @ Z Z$ & $P b^{0} @ A C$ \\
\hline Binding energy, eV & 0.199 & 0.246 & 0.043 & 0.066 & 0.038 & 0.057 & 0.039 & 0.057 & 0.039 & 0.057 \\
\hline Charge on Pb & 0.191 & 0.235 & 0.000 & 0.017 & -0.022 & -0.019 & -0.024 & -0.021 & -0.026 & -0.024 \\
\hline Solvation energy, eV & - & - & -0.573 & -0.604 & -0.764 & -0.808 & -0.789 & -0.835 & -0.816 & -0.865 \\
\hline HOMO, Hartree & -0.117 & -0.1179 & -0.125 & -0.1244 & -0.132 & -0.1315 & -0.133 & -0.1325 & -0.134 & -0.1337 \\
\hline LUMO, Hartree & -0.089 & -0.0863 & -0.092 & 0.0851 & -0.094 & -0.0876 & 0.094 & -0.0880 & -0.095 & -0.0884 \\
\hline$E_{\text {номо-LUмо }}, \mathrm{eV}$ & 0.739 & 0.860 & 0.900 & 1.069 & 1.027 & 1.194 & 1.046 & 1.212 & 1.067 & 1.232 \\
\hline Electrophilicity, eV & 10.725 & 8.981 & 9.721 & 7.604 & 9.281 & 7.448 & 9.220 & 7.433 & 9.155 & 7.415 \\
\hline Hardness, eV & 0.369 & 0.430 & 0.450 & 0.534 & 0.513 & 0.597 & 0.523 & 0.606 & 0.533 & 0.616 \\
\hline
\end{tabular}


We can argue that the modification of the $\mathrm{Pb}$ adsorption mechanism is due to a solventmediated screening of the interaction between $\mathrm{Pb}$ adatoms with both ZZ-GQD and AC-GQD. Such phenomenon may be caused by the energy penalty that must be paid by the interacting system to be partially desolvated. Indeed, as we can see from Table 4, the increase in dielectric constant of the solvent gives rise to lowering of the solvation energy for both

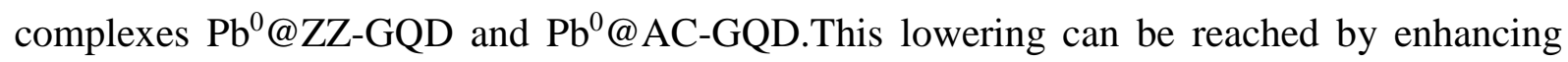
the $\mathrm{Pb}^{0}$-solvent interaction. In other words, the desolvation penalty incurred after interaction between $\mathrm{Pb}^{0}$ and GQDs weakens the binding ability of GQD to $\mathrm{Pb}^{0}$. It should be noted that the overall decrease in interaction strength between neutral lead atom and both GQDs implies two important results (see also Table 4): (i) solvent-induced HOMO-LUMO band gap widening and (ii) a decrease in electrophilicity index with increasing the dielectric constant of solvent. As a consequence, the global hardness gradually increases with the dielectric constant of the solvent, reaching the maximal value for water. This infers that the $\mathrm{Pb}^{0} @ Z Z-G Q D$ and $\mathrm{Pb}^{0} @ \mathrm{AC}-\mathrm{GQD}$ systems become more stable and less reactive when being immersed in the solvent with high polarity.

As may be expected, the changes in electronic properties of the $\mathrm{Pb}^{0} @ Z Z-G Q D$ and $\mathrm{Pb}^{0} @ \mathrm{AC}-$ GQD systems in the presence of the solvent will lead to variations in their absorption spectra. Let's now take a closer look at the absorption curves of Pb ${ }^{0} @ Z Z-G Q D$ and Pb $^{0} @ A C-G Q D$ in different media (see Figure 6).
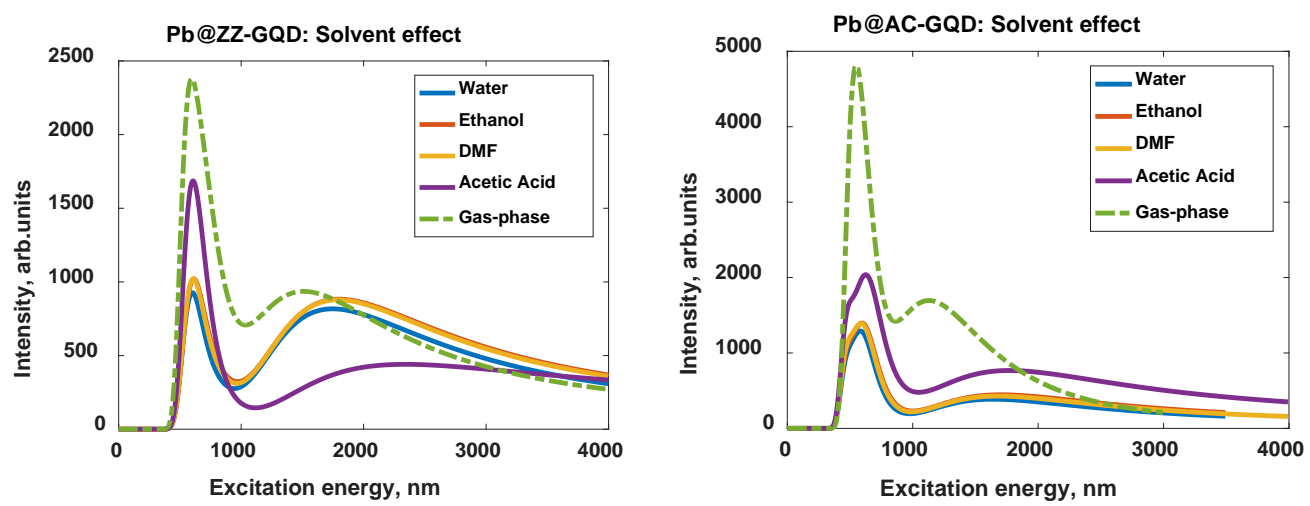

Figure 8. Absorption spectra of the GQDs after complexation with $\mathrm{Pb}^{0}$ obtained in different dielectric media by using the PCM/TD-DFT/B3LYP/6-31G calculations of excited transitions: (left panel) zigzag edge termination and (right panel) armchair edge termination

A general trend of these curves is the drop of absorption intensity with increasing the dielectric constant of the solvent according to the following order: gas-phase $>$ acetic acid $>$ ethanol> DMF > water. In the gas phase, the absorption spectrum of the $\mathrm{Pb}^{0} @ \mathrm{ZZ}-\mathrm{GQD}$ is 
composed of a wide dominant band centred at $\sim 729 \mathrm{~nm}$ and a broad emission ranging from 1000 to $4000 \mathrm{~nm}$. As shown in Fig. 9a, the first band is mainly associated with the $\mathrm{HOMO} \rightarrow \mathrm{L}+6$ transition $(729 \mathrm{~nm})$ with oscillator strength $f=0.0259$, while some contribution of the transition at $555 \mathrm{~nm}$ with total oscillator strength of 0.024 to the short-wavelength side of the main peak is coming from the multi-level excited transitions (HOMO-2 $\rightarrow$ LUMO, HOMO- $\rightarrow$ LUMO+1, HOMO- $\rightarrow$ LUMO, HOMO $\rightarrow$ LUMO+10). The detailed description of the existing transitions and their origin is listed in Dataset S3 (Electronic Supplementary Information\#2). The broad absorption band centred at $1468 \mathrm{~nm}$ is originating from a mix of two components: $\mathrm{HOMO} \rightarrow \mathrm{LUMO}+1$ and $\mathrm{HOMO} \rightarrow \mathrm{LUMO}+3$, respectively. Due to the strong interaction between a neutral $\mathrm{Pb}$ adatom and a ZZ-GQD, the absorption features are attributed to the transitions to/or from highly hybridized molecular orbitals (which are shared by both $\mathrm{Pb}$ adatom and ZZ-GQD).

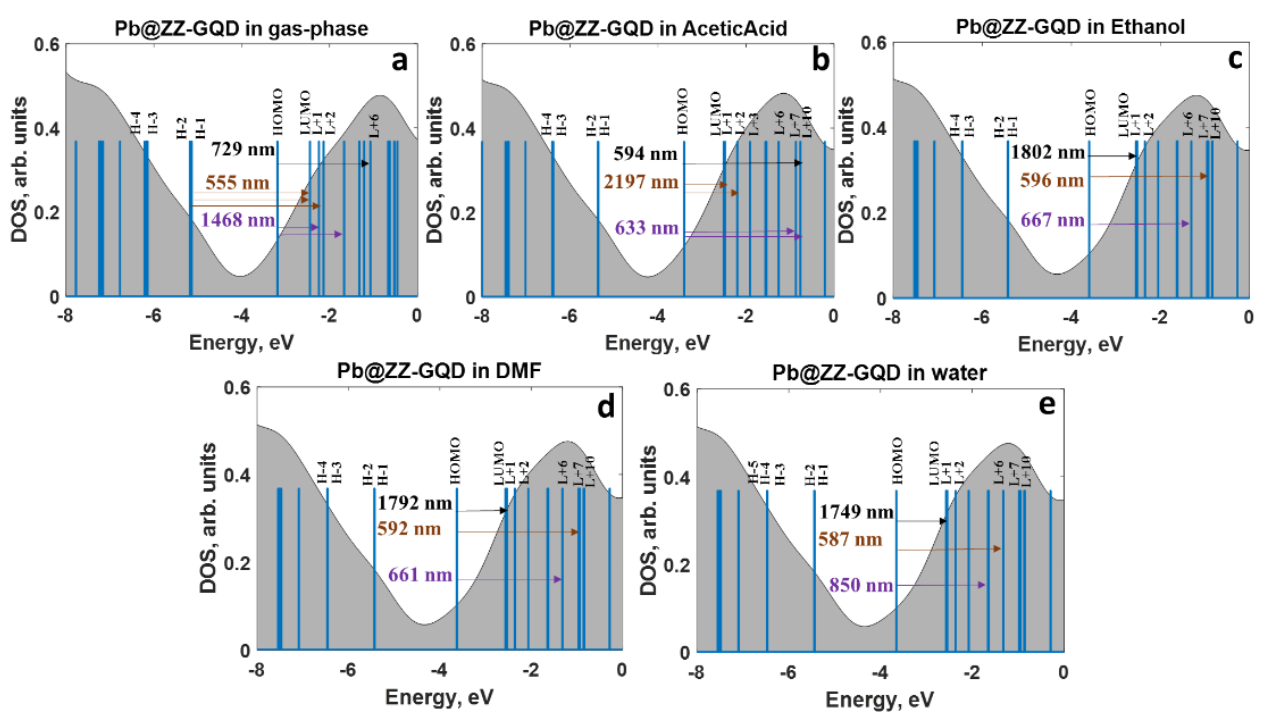

Figure 9. DOS of zigzag-edged GQDs after complexation with $\mathrm{Pb}^{0}$ (shaded area) immersed in

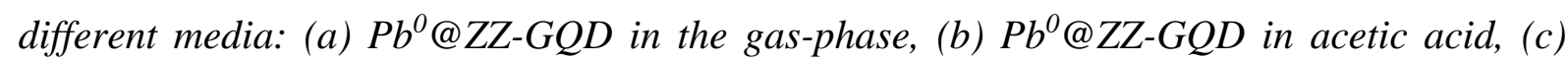
Pb@ZZ-GQD in the ethanol, (d) Pb $b^{0} @ Z Z-G Q D$ in the DMF, and (e) Pb $b^{0} @ Z Z-G Q D$ in the water. Blue vertical lines denote the molecular orbitals. The excited transitions between available occupied and unoccupied electronic levels are shown by black arrowed lines.

It is interesting to note that after complexation of $\mathrm{Pb}$ with ZZ-GQD in the acetic acid (solvent with small polarity and low value of the dielectric permittivity), the hybrid orbitals become less involved in excited transitions. To be more exact, only the HOMO level (which is almost localized on $\mathrm{Pb}$ adatom, see Figure S5, Electronic Supplementary Information\#2) takes part in the transitions (see Figure 9b and Dataset S3, Electronic Supplementary Information\#2). The absorption spectra in this case consist of three most intensive features. The band at about 594 
$\mathrm{nm}$ is attributable to the transition of the electrons HOMO to LUMO+10 (which is delocalized over the surface of GQD). The excited transitions across the HOMO-LUMO gap are schematically shown in Fig. 9b for other two absorption wavelengths at 2197 and $693 \mathrm{~nm}$, respectively. As was mentioned before, the multi-band absorption features of the $\mathrm{Pb}^{0} @ \mathrm{ZZ}-$ GQD are highly sensitive to the solvent polarity, which provides further support for that the quenching of the absorption intensity of the two observed bands is mainly attributed to the solvent-induced screening of the attractive interaction between the $\mathrm{Pb}$ adsorbate and $\mathrm{ZZ}$ GQD. This is particularly the case of $\mathrm{Pb}^{0} @ Z Z-G Q D$ immersed in ethanol, DMF and water, where significant weakening of the interaction strength between $\mathrm{Pb}$ and ZZ-GQD leads to blue-shift of the absorptions bands in comparison to those for acetic acid (Fig. 9, c-e). The solvent effect is significant also for the absorption properties of the armchair-edged GQDs interacted with neutral $\mathrm{Pb}$ adatom. The nature of the excited transitions in armchair-edged GQDs after complexation with $\mathrm{Pb}^{0}$ is described in the text of the Electronic Supplementary Information \#1 (Section 1).

\subsubsection{Validation of the calculation method for estimating the weak interaction between neutral HMs and differently shaped GQDs}

In the absence of reliable experimental benchmark data the correct estimating of the binding ability of the GQDs to heavy metals is still a challenge. Therefore, it is very important to use different functionals and levels of theory to study this interaction, to collect more comparative data and to validate affordable methods for improving the benchmark parameters describing the HM@GQD complexation. In many cases (van der-Waals interaction, weak $\pi$ - $\pi$ interaction) B3LYP functional cannot be used to accurately model the interactions between two large systems, especially the weak physisorption. On the other hand, because the complexes composing of finite-sized GQD and single atom are comparatively small, they can be studied accurately using even B3LYP method, as was predicted for hydrogen adsorption onto graphene ${ }^{47}$, for interaction between Pt and nanometer-size graphene ${ }^{48}$ and for adsorption of $\mathrm{Mn}$ (and other transition metals) on pristine and defected graphene ${ }^{49}$. Therefore, in the present paper we are mainly focusing on results of the B3LYP level of theory. Furthermore, the correctness of the B3LYP/6-31g method for study of the interaction between heavy metals and graphene was also confirmed by independent VASP calculations using periodical boundary conditions (DFT-D3 method of Grimme, GGA-PBE) ${ }^{19}$. We revealed that the main trends (order of the binding energy, charge transfer, binding height and binding positions of the HMs on extended graphene) are in broad agreement with those determined for GQDs by B3LYP/6-31g method. The B3LYP/6-31G level of theory has then been validated as that 
providing the good agreement with the results of the supercell VASP method (which describes the van der Waals interactions).

Nevertheless, to verify the propriety of our calculation we also performed additional comparative calculations using dispersion-corrected M06-2X DFT ${ }^{50,51}$ and PBE-D3 DFT ${ }^{52}$ functionals with the extended 6-31G(d,p) basis set. It should be mentioned that among both of them only the PBE-D3 method takes into account explicitly London dispersion forces (or van der Waals forces) by using an additional term (which is proportional to $R^{-6}$ ), whereas the M06-2X functional includes implicitly some modified parameters related to Hartree-Fock exchange interaction.

A comparative analysis of the results obtained using different methods indicates that the geometry (binding site, binding height) is not very sensitive to functional choice (Figure S6 and Figure S7, Electronic Supplementary Information\#2).As one can see from the Figures S8S10 (Electronic Supplementary Information\#2), the calculated binding energies exhibit same trends: increasing the dielectric permittivity leads to increase in the binding energy of $\mathrm{Cd}(\mathrm{Hg})$ and decrease in the binding energy of the neutral $\mathrm{Pb}$ atom. It is a remarkable finding that the binding-energy difference between M06-2X (PBE-D3) and B3LYP is significantly larger for Cd (Hg)@GQDs complexes, than for Pb@GQDs. It is obvious that M06-2X and PBE-D3 functionals strongly overestimated the binding energies of the $\mathrm{Cd}, \mathrm{Hg}$ and $\mathrm{Pb}$ in all considered solvents compared to B3LYP functional. Furthermore, according to M06-2X and PBE-D3 calculations a change of the dielectric permittivity of the medium switches the nature of $\mathrm{Cd}$ (Hg) adsorption from physisorption to chemisorption. This may be associated with the fact that the dispersion-corrected DFT methods are not appropriate, to some extent, to treat correctly the dispersion interactions in the presence of solvent.

We then compare the dielectric constant-dependent behaviour of the HOMO-LUMO gap of the considered complexes calculated by different methods. For all considered metals, the M06-2X and PBE-D3 functionals predicted that a trend for HOMO-LUMO gaps with increase in the dielectric permittivity is in qualitative agreement with B3LYP results (see Figs. S11-S13, Electronic Supplementary Information\#2). PBE-D3 gives smaller HOMOLUMO gaps of all interacting complexes in comparison to B3LYP results, while M06-2X significantly overestimated the value of the HOMO-LUMO gaps.Finally, it must be noticed that the absorption spectra of GQDs before and after complexation with HMsare very sensitive to the choice of the functional (Figures S14-S18, Electronic Supplementary Information\#2), which is in line with the solvent-induced changes in the DOS structure (HOMO-LUMO gap). For this reason, the spectra calculated by using TD-PBE-D3/6- 
$311 \mathrm{~g}(\mathrm{~d}, \mathrm{p})$ method are red-shifted in the comparison to those calculated by TD-B3LYP/6-31g, while TD-M06-2X/6-311g(d,p) calculation results in the blue-shifted spectra.In general there is good agreement between the calculated spectra regarding the solvent role in the excited transitions in pristine GQDs and Hg@GQDs (Figures S14 and S16). On the other hand, as can be seen from the Figures S15, S17 and S18 the absorption spectra of the Cd@GQDsand $\mathrm{Pb} @ G Q D s$ obtained byTD-B3LYP/6-31g in the presence of the solvent differ, to some extent, from those calculated by TD-M06-2X/6-311g(d,p) and TD-PBE-D3/6-311g(d,p). It is important to note that dispersion-corrected methodgives enhanced values of the oscillator strengths of the main electronic transitions in Pb@GQD complexes immersed in solvents with high polarity in comparison to TD-B3LYP/6-31g. The discrepancy between the absorption spectra calculated by different methodsarises becauseM06-2X and PBE-D3functionalsyield overestimated values of binding energies and charge transfer and, therefore, they poorly describe the dominant electronic transitionsin the interacting systems considered in this paper.

\subsection{Solvent-mediated interaction between GQDs and divalent HM ions: geometry, electronic and optical properties.}

In the preceding section we have shown that the solvent significantly affects the interaction strength of neutral heavy metal adatoms with zigzag-/armchair-edged GQDs. For such systems we predicted how the solvent effect manifests itself in their electronic structure and excited states. Nevertheless, in most cases the experimental determination of heavy metals in liquids implies that the heavy metal species considered are in charged state (usually divalent ions). In particular, the sensing mechanismunderlying the selective recognition of HMs byUV-vis absorption spectroscopy or fluorescencespectroscopy is based on collecting of various optical responses to specific interactions between heavy metal ions and GQDs.Bearing in mind the main idea of this work, the divalent $\mathrm{HM}$ ions $\left(\mathrm{Cd}^{2+}, \mathrm{Hg}^{2+}, \mathrm{Pb}^{2+}\right)$ complexed with ZZ-GQDs and AC-GQDs will be subjected to a detailed examination of how dielectric medium with varying permittivity influences the binding affinity, electronic and optical properties of the interacting systems.

\subsubsection{Cd ${ }^{2+} @ G Q D$}

The behaviour of the divalentHM ions is not similar tothat of neutral speciesonce adsorped on the surface of GQDs. ${ }^{19}$ In particular, it wasshownthatthe adsorption mechanism of charged ions is mainly governed by chemisorption, which involves a strong chemical reaction between the GQD and metal ions.Regarding the $\mathrm{Cd}^{2+}$ case, we anticipate thatthe bridge site isthe most stable configurationunder vacuum conditions for chemisorption of $\mathrm{Cd}^{2+}$ on both ZZ-GQD and AC-GQD, with the binding energies of $11.663 \mathrm{eV}$ and $11.575 \mathrm{eV}$, respectively. From Table 5, 
one can see that thebinding energy ofC $\mathrm{d}^{2+}$ drastically decreases with increasing the dielectric constant of the solvent, reaching the lowest values for the solvent with the highest polarity. In addition, we noticed the changes in the $\mathrm{Cd}^{2+}$ adsorption site whenever the interactionoccurred in solvent with different dielectric permittivity (see Table. S6, Electronic Supplementary Information\#2).Although the gas-phase calculations suggest that the binding energy of $\mathrm{Cd}^{2+}$ on ZZ-GQD is slightly larger than that on AC-GQD, the interaction strength in the solvent demonstrates an opposite trend, being always larger in the case of $\mathrm{Cd}^{2+}$ on AC-GQD. Such a decreasing tendencyindicates solvent-mediated weakening of the chemical bonding between $\mathrm{Cd}^{2+}$ and GQDs and even changing of the chemisorption to physical adsorption when the dielectric constant of the solvent is increased. The obtained results can be understood in terms of the so-called cation- $\pi$ interaction. ${ }^{53}$ Indeed, it was shownthat solvents can modulate the binding affinity of the electron-rich $\pi$ system to metals ions. This is mainly due to the fact that the energy gainfromthe interaction between charged ion and $\pi$ system is compensated, to some extent, by the loss of solvation energy.In line with this we also found that thedesolvationaccompanying the $\mathrm{Cd}^{2+}$ adsorptionon AC-GQD in the presence of highly polar solvents is slightly larger as that accompanying the adsorption of $\mathrm{Cd}^{2+}$ on ZZ-GQD (Table 5).Such changes of adsorption configurations induced by solvents agree well with an enhancement of the stability of the interacting systems. In particular, this is confirmed by a dramatic decrease in the electrophilicity index, increase in the global hardness and HOMOLUMO gap widening, as is shown in Table 5.

Table 5. Computed parameters of zigzag- and armchair-edged GQDs after complexation with $\mathrm{Cd}^{2+}$ in different media

\begin{tabular}{|c|c|c|c|c|c|c|c|c|c|c|}
\hline \multirow[t]{2}{*}{ Parameter } & \multicolumn{2}{|c|}{$\begin{array}{c}\text { Gas-phase } \\
\varepsilon=1\end{array}$} & \multicolumn{2}{|c|}{$\begin{array}{c}\text { Acetic acid } \\
\varepsilon=6.2528\end{array}$} & \multicolumn{2}{|c|}{$\begin{array}{c}\text { Ethanol } \\
\varepsilon=24.852\end{array}$} & \multicolumn{2}{|c|}{$\begin{array}{c}\text { DMF } \\
\varepsilon=37.219\end{array}$} & \multicolumn{2}{|c|}{$\begin{array}{c}\text { Water } \\
\varepsilon=78.355\end{array}$} \\
\hline & $C d^{2+}-Z Z$ & $C d^{2+}-A C$ & $C d^{2+}-Z Z$ & $C d^{2+}-A C$ & $C d^{2+}-Z Z$ & $C d^{2+}-A C$ & $C d^{2+}-Z Z$ & $C d^{2+}-A C$ & $C d^{2+}-Z Z$ & $C d^{2+}-A C$ \\
\hline Binding energy, eV & 11.663 & 11.575 & 0.751 & 0.827 & 0.130 & 0.178 & 0.144 & 0.192 & 0.104 & 0.259 \\
\hline Charge on Cd & 0.22 & 0.26 & 1.71 & 1.72 & 2.03 & 2.02 & 2.05 & 2.03 & 2.07 & 2.06 \\
\hline Solvation energy, eV & - & - & -5.103 & -5.321 & -6.824 & -7.032 & -7.101 & -7.312 & -7.340 & -7.660 \\
\hline$E_{\text {номо-LUмо }}, \mathrm{eV}$ & 0.665 & 0.781 & 0.977 & 1.244 & 1.404 & 1.586 & 1.595 & 1.780 & 1.774 & 2.009 \\
\hline Electrophilicity, eV & 156.519 & 134.435 & 35.483 & 29.140 & 17.218 & 15.453 & 14.204 & 12.909 & 12.111 & 10.571 \\
\hline Hardness, eV & 0.332 & 0.390 & 0.488 & 0.622 & 0.702 & 0.793 & 0.797 & 0.890 & 0.887 & 1.004 \\
\hline
\end{tabular}

To illustrate both, the role of $\mathrm{Cd}^{2+}$ ion in electronic transitions and the solvent effect on the optical properties of $\mathrm{Cd}^{2+} @ \mathrm{GQD}$, let's consider the absorption spectra of the interacting systems dispersed in different media (Fig. 10). Comparing the different spectra of the $\mathrm{Cd}^{2+} @ Z Z-G Q D$ (left panel, Figure 10), one can conclude that (i) on increasing the dielectric constant, the absorption peak undergoes blue-shift and (ii) aconsiderable weakening of the 
charge transfer between adsorbate and ZZ-GQD results inan intense absorption in the visible range.
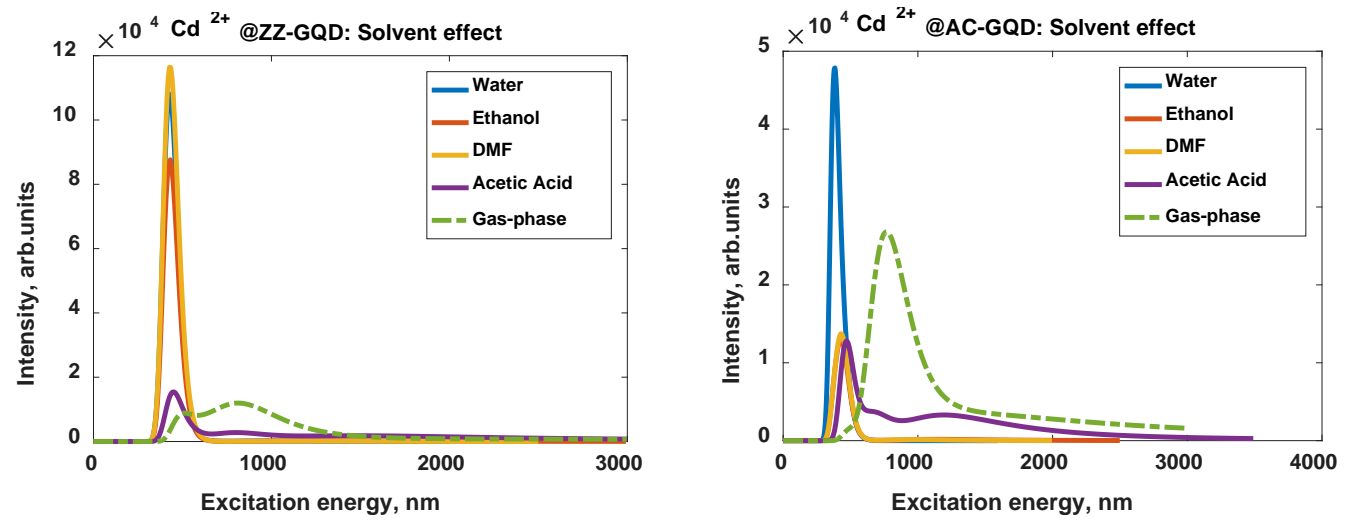

Figure 10. Absorption spectra of the GQDs after complexation with $\mathrm{Cd}^{2+}$ obtained in different dielectric media by using the PCM/TD-DFT/B3LYP/6-31G calculations of excited transitions: (left panel) zigzag edge termination and (right panel) armchair edge termination.

In the absorption curve of the $\mathrm{Cd}^{2+} @ Z Z-G Q D$ (the gas-phase case) three major peaks are apparent at $489 \mathrm{~nm}$ (oscillator strength $f=0.1804), 632 \mathrm{~nm}(f=0.1198)$ and $832 \mathrm{~nm}(f=0.2318)$, respectively (see also Fig. 11 a).The absorption feature at $489 \mathrm{~nm}$ is primarily due to the overlapping of the two electronic transitions from the double degenerate lowest occupied levels to the unoccupied levels: HOMO-1 $\rightarrow$ LUMO+2 (59\%) and HOMO $\rightarrow$ LUMO+1 (37 $\%)$. Among these molecular orbitals we found that the contribution of $\mathrm{Cd}^{2+}$ to HOMO-1 is the largest and is estimated to be approximately 91 \% (Figure S19, Electronic Supplementary Information\#2). In addition, a negligible small contribution of $\mathrm{Cd}^{2+}(7 \%)$ to LUMO is also observed (Figure S19, Electronic Supplementary Information \#2). The wave functions of other orbitals are mainly delocalized over the surface of the ZZ-GQD. The second absorption peak at $632 \mathrm{~nm}$ reflects the single transition from GQD-related HOMO-5 to LUMO (with slight hybridization). Finally, the most intensive peak, which is observed at $832 \mathrm{~nm}$, corresponds to two interband transitions: HOMO-2 $\rightarrow$ LUMO (90 \%) and HOMO $\rightarrow$ LUMO+1 (11\%). As can be seen from the Fig. 10 (left panel) and Fig. 11 b, increasing the dielectric constant of the environment from 1 (gas-phase case) to 6.25 (acetic acid case) leads to significant changes in absorption spectra and, as a consequence, to the appearance of the new transitions. In particular, the spectrum is composed of the dominant feature at $444 \mathrm{~nm}$ and the weak absorption bands at 543 and $817 \mathrm{~nm}$. Regarding the origin of the $444 \mathrm{~nm}$ peak, the most likely absorption transitions are HOMO-9 $\rightarrow$ LUMO (66 \%) and HOMO-1 $\rightarrow$ LUMO+2 (15\%). It is important to note that $\mathrm{Cd}^{2+}$ contributes to these levels in different 
ways: 4 \% in HOMO-9 and 77 \% LUMO (Fig. S19, Electronic Supplementary Information \#2).
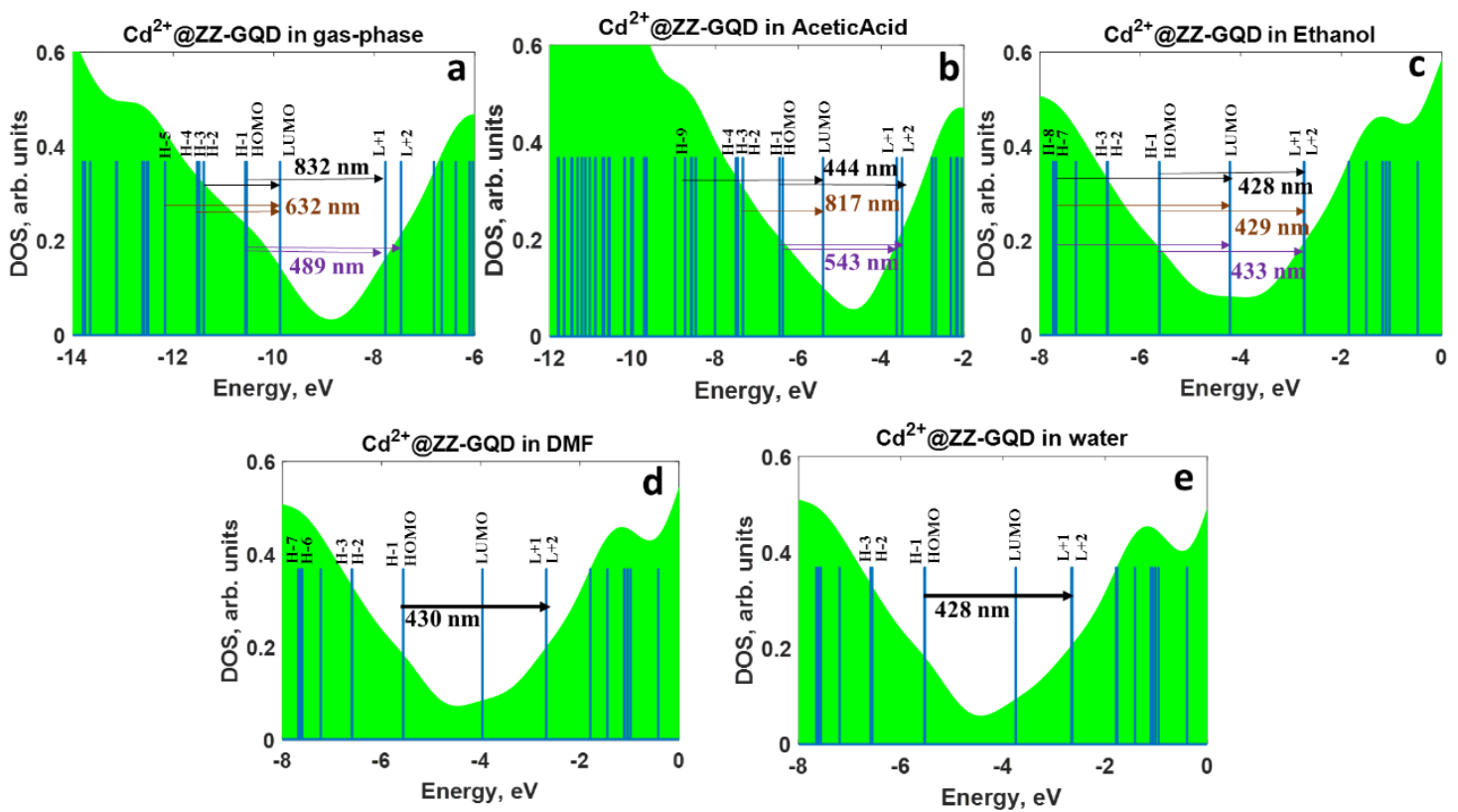

Figure 11. DOS of zigzag-edged GQDs after complexation with $\mathrm{Cd}^{2+}$ (shaded area) immersed

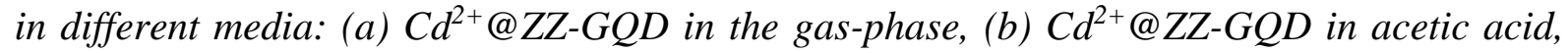

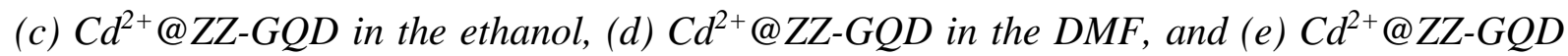
in the water. Blue vertical lines denote the molecular orbitals. The excited transitions between available occupied and unoccupied electronic levels are shown by black arrowed lines.

The weak band at $543 \mathrm{~nm}$ can be ascribed to the following transitions: HOMO- $\rightarrow$ LUMO +2 $(35 \%)$ and $\mathrm{HOMO} \rightarrow \mathrm{LUMO}+1(66 \%)$. A small contribution of $\mathrm{Cd}^{2+}$ to HOMO-1 (10 \%) and LUMO-1 (3\%) is revealed. At the same time the peak at $817 \mathrm{~nm}$ is mainly caused by transition from the HOMO-2 to LUMO. Moving from the acetic acid to ethanol, we noticed significant enhancement of the absorption intensity in visible. This is due to the appearance of the set of overlapped peaks with similar energies (428 nm, $429 \mathrm{~nm}$, and $433 \mathrm{~nm})$ and high oscillator strengths (Fig. $11 \mathrm{c}$ ). The most intensive component at $428 \mathrm{~nm}$ is arising from the configuration of the three most likely transitions, including HOMO-7 $\rightarrow$ LUMO (14 \%), $\mathrm{HOMO}-1 \rightarrow \mathrm{LUMO}+2(42 \%)$ and $\mathrm{HOMO} \rightarrow \mathrm{LUMO}+1(41 \%)$. It should be mentioned that only LUMO is strongly localized at $\mathrm{Cd}^{2+}$, while other orbitals are delocalized in nature (Figure S19, Electronic Supplementary Information \#2). The enhanced transition probability can be explained by the fact that double degenerate HOMO/HOMO-1 and LUMO+1/LUMO+2 are involved into the transitions. Another absorption feature at $429 \mathrm{~nm}$ 
corresponds to similar transitions, but in this case the degenerate HOMO-8 level participates instead of the HOMO-7. The last one is mainly related to HOMO-7 $\rightarrow$ LUMO. The Cd-related LUMO does not contribute to the excited states (Figure S19, Electronic Supplementary Information \#2) with further increase in the dielectric permittivity of the solvent (case of high polarity: DMF and water). For polar solvents, only transitions between double degenerate HOMO/HOMO-1 and LUMO+1/LUMO+2 are dominant (Fig. $11 \mathrm{~d}$ and e).

Moving from Cd2+@ZZ-GQD to the Cd ${ }^{2+} @ A C-G Q D$ we see (right panel, Figure 10 and Figure S2, Electronic Supplementary information \#1) that similarly to the previous case the increase in the dielectric constant of the environment causes a significant HOMO-LUMO gap widening, followed by the blue-shift of the absorption peak. More details of the nature of the excited transitions in $\mathrm{Cd}^{2+} @ \mathrm{AC}-\mathrm{GQD}$ are described in Electronic Supplementary Information \#1 (Section 2).

\subsection{2. $\mathrm{Hg}^{2+} @ G Q D$}

Here the binding ability of the zigzag- and armchair-edged GQDs to divalent mercury ions in different media is in focus.Our results indicate that the $\mathrm{Hg}^{2+}$ ion tends to bind more frequently to on-top site of the ZZ-GQDs in all considered media, except the water (see the Table S7, Complementary Materials). While the interaction between AC-GQDs and $\mathrm{Hg}^{2+}$ ions alwaysleads to stabilization of the mercury ions at the hollow site.Absorption $\mathrm{of}^{\mathrm{Hg}^{2+}}$ ion under vacuum conditions is accompanied by a charge transfer of $\sim 1.9$ e- from the ZZGQD/AC-GQD to the mercury species (Table 6). In this way, $\mathrm{Hg}^{2+}$ adsorbate acts as an effective electron acceptor. Moving from the gas-phase case to the acetic acid it is obvious that $\mathrm{Hg}$ becomes more positively charged, thereby indicating the reduction of the charge transfer due to the solvent effect. It is interesting to note that as the polarity of the solvent becomes increasingly high, the vanishing of the charge transfer becomes more pronounced, which weakens the interaction between ZZ-GQD/AC-GQD and $\mathrm{Hg}^{2+}$ ion. This trend is also seen in the dependence of the binding energy on the dielectric constant: as the dielectric constant is increased, the binding energy decreases, reaching the minimal values for ethanol, DMF and water. Similarly to the $\mathrm{Cd}^{2+}$ case, this can be explained by the modification in how electron-rich $\pi$ system of GQD interacts with the positively charged cation in the presence of the polar solvents. ${ }^{54}$ In this context, the solvation energies are found to be larger for interacted systems immersed in solvents with high polarity. It should be also mentioned that the binding energy of $\mathrm{Hg}^{2+}$ ion in the whole range of the dielectric constant values is unexpectedly greater than the binding energy of $\mathrm{Cd}^{2+}$. Furthermore, $\mathrm{Hg}^{2+}$ has the lower positive charge after interaction with both ZZ-GQDs and AC-GQDs in the gas-phase compared to $\mathrm{Cd}^{2+}$. From the 
theoretical point of view, the metal species with smaller ionic radius should bind with GQDs in charge transfer manner. ${ }^{55}$ Contrarily, as the ionic radius of the metal species becomes larger the electrostatic interaction should start to dominate. Since $\mathrm{Hg}^{2+}$ has higher ionic radius than $\mathrm{Cd}^{2+}$ (102 pm vs. $95 \mathrm{pm}$ ), one would expect a higher charge transfer from GQDs to $\mathrm{Cd}^{2+}$. But our results show the opposite trend, indicating that the charge transfer is main factor, which is responsible for the strong interaction between $\mathrm{Hg}^{2+}$ and GQDs, while the chemisorption of the $\mathrm{Cd}^{2+}$ ions is more electrostatic in nature. According to the literature, such nontrivial behaviour of the mercury ions interaction with other molecules can be explained by several factors, including (i) relativistic effects for $\left[\mathrm{Hg}^{2+}\right]^{56}$ and (ii) a presence of $5 f$ inner orbitals for $\mathrm{Hg}^{2+}$, which are mainly responsible for the enhanced charge accepting ability (these orbitals are absent in the case of $\left.\mathrm{Cd}^{2+}\right) \cdot{ }^{57}$ We noticed also that in both cases $\left(\mathrm{Hg}^{2+} @ Z Z-G Q D s\right.$ and $\left.\mathrm{Hg}^{2+} @ A C-G Q D s\right)$, the electrophilicity index dramatically decreases in the presence of the solvent with high polarity. It can be caused by changes in the chemical potential and HOMO/LUMO energy positions. Indeed, as is seen in Table 6 HOMO and LUMO energy levelsmove upward in the solvent phase in comparison to the gas-phase.The observed HOMO-LUMO gap widening and theincrease in the global hardness are in line with solventinduced binding energy reduction.

Table 6. Computed parameters of zigzag-and armchair-edged GQDs after complexation with $\mathrm{Hg}^{2+}$ in different media

\begin{tabular}{|c|c|c|c|c|c|c|c|c|c|c|}
\hline \multirow[t]{2}{*}{ Parameter } & \multicolumn{2}{|c|}{$\begin{array}{c}\text { Gas-phase } \\
\varepsilon=1 \\
\end{array}$} & \multicolumn{2}{|c|}{$\begin{array}{c}\text { Acetic acid } \\
\varepsilon=6.2528\end{array}$} & \multicolumn{2}{|c|}{$\begin{array}{c}\text { Ethanol } \\
\varepsilon=24.852 \\
\end{array}$} & \multicolumn{2}{|c|}{$\begin{array}{c}\text { DMF } \\
\varepsilon=37.219\end{array}$} & \multicolumn{2}{|c|}{$\begin{array}{c}\text { Water } \\
\varepsilon=78.355\end{array}$} \\
\hline & $\mathrm{Hg}^{2+}-\mathrm{ZZ}$ & $\mathrm{Hg}^{2+} \mathrm{AC}$ & $\mathrm{Hg}^{2+}-\mathrm{ZZ}$ & $\mathrm{Hg}^{2+} \mathrm{AC}$ & $\mathrm{Hg}^{2+}-\mathrm{ZZ}$ & $\mathrm{Hg}^{2+} \mathrm{AC}$ & $\mathrm{Hg}^{2+}-\mathrm{ZZ}$ & $\begin{array}{c}\mathrm{Hg}^{2+}- \\
\mathrm{AC}\end{array}$ & $\mathrm{Hg}^{2+}-\mathrm{ZZ}$ & $\mathrm{Hg}^{2+}-\mathrm{AC}$ \\
\hline Charge on atom & 0.09 & 0.09 & 0.44 & 0.12 & 1.85 & 2.06 & 2.01 & 2.06 & 2.04 & 2.07 \\
\hline Solvation energy, eV & - & - & -4.431 & -4.308 & -5.494 & -5.704 & -5.776 & -6.003 & -6.101 & -6.327 \\
\hline LUMO, Hartree & -0.3646 & -0.3643 & -0.2225 & -0.2297 & -0.1834 & -0.1809 & -0.1731 & -0.1712 & -0.1616 & -0.1607 \\
\hline$E_{\text {номо-LUMо }}, \mathrm{eV}$ & 0.666 & 0.944 & 0.627 & 0.244 & 0.683 & 0.813 & 0.848 & 1.016 & 1.082 & 1.237 \\
\hline Electrophilicity, eV & 157.895 & 114.258 & 64.604 & 166.427 & 41.643 & 34.943 & 31.065 & 26.277 & 22.540 & 20.132 \\
\hline Hardness, eV & 0.333 & 0.472 & 0.313 & 0.122 & 0.341 & 0.406 & 0.424 & 0.508 & 0.541 & 0.618 \\
\hline
\end{tabular}

To understand more deeply the unusual behavior of the $\mathrm{Hg}^{2+} @$ GQDs in the presence of a solvent, we undertook calculations of the absorption spectra and the electronic structure. A summary of the obtained results is provided byFigures 12 and 13. As we can see from the Figure 12, the changes in the dielectric permittivity of the environment leads to a significant modification of the absorption spectra; these changes manifest themselves in the dramatic decrease of the absorption intensity of both $\mathrm{Hg}^{2+} @ Z Z-G Q D s$ and $\mathrm{Hg}^{2+} @ A C-G Q D s$ dispersed in solvents with high polarity (mainly ethanol, DMF and water). While the electronic 
transitions in the presence of the acetic acid are also affected, but to a lesser degree. In this case, we can only observe slight shift of the absorption peaks and the redistribution of the electron charge density following different electronic transitions. Analysing the absorption curve of the $\mathrm{Hg}^{2+} @ Z Z-G Q D$ in the gas-phase (Figure 13a), we noticed that the lowest excited transition between HOMO and LUMO is forbidden, and $\mathrm{Hg}^{2+}$ does not contribute significantly to the energy levels, which are involved in allowed transitions. Overall, three main absorption peaks at 466, 625 and $820 \mathrm{~nm}$ are observed. The most probable transitions for the 446 nm-peak are HOMO-2 $\rightarrow$ LUMO (12 \%) and HOMO $\rightarrow$ LUMO+1 (85 \%) transitions, while the HOMO-5 $\rightarrow$ LUMO is mainly responsible for the appearance of the 625 nm-peak. The absorption feature at the $820 \mathrm{~nm}$ can be ascribed to the HOMO-2 $\rightarrow$ LUMO (88 $\%)$ and $\mathrm{HOMO} \rightarrow \mathrm{LUMO}+1(12 \%)$ transitions. We believe that an enhanced absorption intensity of the $\mathrm{Hg}^{2+} @ Z Z-G Q D$ in this case can be reasonably explained by the nature and composition of the orbitals participating in the electronic transitions. It's very probable, and maybe inevitable that the weak contribution of mercury to the main orbitals promotes electronic transitions with high oscillator strengths between energy levels delocalized over the planar structure of the ZZ-GQD. Surprisingly, the absorption spectrum in acetic acid undergoes significant changes. In particular, the short-wavelength part of this spectrum reduces in intensity compared to its counterpart in the gas-phase, while the long-wavelength shoulder increases in intensity. In fact, the bands at 509, 645 and $862 \mathrm{~nm}$ form the absorption pattern of the $\mathrm{Hg}^{2+} @ Z Z-G Q D$ in acetic acid. We assign the lowest wavelength absorption peak at $509 \mathrm{~nm}$ to the following transitions: HOMO-1 $\rightarrow$ LUMO+1 (39 \%) and $\mathrm{HOMO} \rightarrow \mathrm{LUMO}+2(45 \%)$. It is worth mentioning that the degenerate HOMO-1/HOMO levels are hybrid in nature (Figure S21, Electronic Supplementary Information\#2), with high percentage of $\mathrm{Hg}^{2+}$ (66 \% / $15 \%$, respectively). In the same way, we assign the absorption peak at $645 \mathrm{~nm}$ to the HOMO-5 $\rightarrow$ LUMO transition. LUMO is also a hybrid orbital with less contribution from $\mathrm{Hg}^{2+}$ (only 17 \%).Finally, we associate the most intensiveabsorption band observed at $862 \mathrm{~nm}$ with HOMO-4 $\rightarrow$ LUMO. Thus, we can conclude that the spectral differences between $\mathrm{Hg}^{2+} @ Z Z-G Q D$ immersed in vacuum conditions and in acetic acid (the solvent with dielectric permittivity as low as 6.25) can be explained by the redistribution of the electron density induced by both Hg effect and solvent effect. Further increase in the dielectric constant of the solvent leads to the increase in the contribution of the $\mathrm{Hg}^{2+}$ to LUMO, followed by a dramatic decrease in the absorption intensity. For polar solvents, the LUMO becomes completely localized at the $\mathrm{Hg}^{2+}$ (Figure S21, Electronic Supplementary Information\#2). Since almost all electronic transitions include LUMO level (Fig.13 c-e), the 
probability of such transitions is very low. Unlike the case of $\mathrm{Cd}^{2+} @ Z Z-G Q D$ in polar solvents, herein the transitions between GQD-related double degenerate occupied and unoccupied energy levels (HOMO-1/HOMO $\rightarrow$ LUMO+1/LUMO+2) are forbidden. That's most likely Hg-related LUMO can trap electrons efficiently, thereby making it impossible for electrons to reach next empty levels (LUMO+1/LUMO+2). See Electronic Supplementary Information \#1 (Section 3) to get more details about the correlation between optical and electronic properties of AC-GQD interacted with $\mathrm{Hg}^{2+}$.
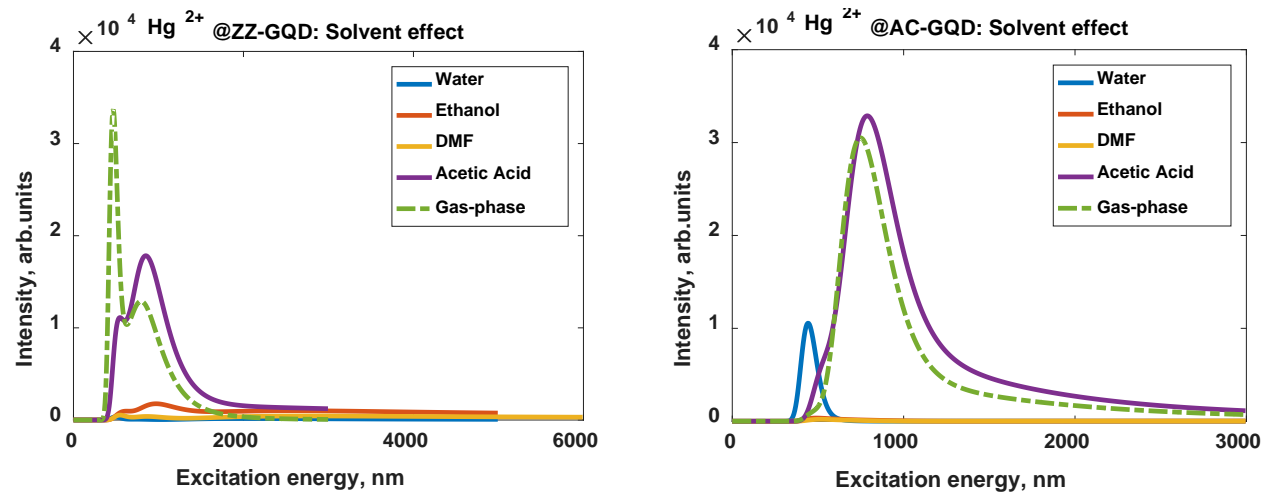

Figure 12. Absorption spectra of the GQDs after complexation with $\mathrm{Hg}^{2+}$ obtained in different dielectric media by using the PCM/TD-DFT/B3LYP/6-31G calculations of excited transitions: (left panel) zigzag edge termination and (right panel) armchair edge termination
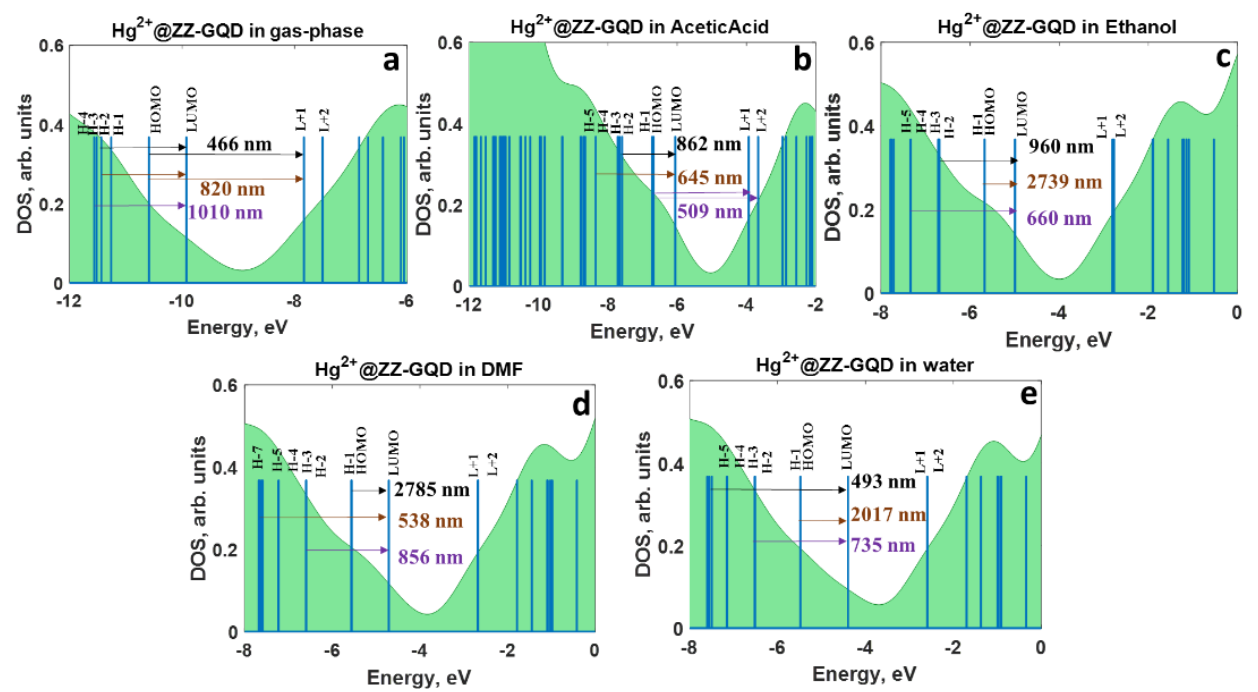

Figure 13. DOS of zigzag-edged GQDs after complexation with $\mathrm{Hg}^{2+}$ (shaded area) immersed in different media: (a) $\mathrm{Hg}^{2+} @ Z Z-G Q D$ in the gas-phase, (b) $\mathrm{Hg}^{2+} @ Z Z-G Q D$ in acetic acid, (c) $\mathrm{Hg}^{2+} @ Z Z-G Q D$ in the ethanol, (d) $\mathrm{Hg}^{2+} @ Z Z-G Q D$ in the DMF, and (e) $\mathrm{Hg}^{2+} @ Z Z-G Q D$ in the water. Blue vertical lines denote the molecular orbitals. The excited transitions between available occupied and unoccupied electronic levels are shown by black arrowed lines. 
In fact, we found experimentalresultswhich are, to some extent, support our findings. In particular, Chakraborti et al. reported that the graphene quantum dot can be used for optical detection of divalent mercury ions in pure aqueous solution by fluorescence chemosensing. ${ }^{24} \mathrm{It}$ was revealed that there is a strong quenching of the optical signal from GQDs after complexation with $\mathrm{Hg}^{2+}$ ions in water. At the same time, no quenching was observed in the case of the interaction between GQDs and $\mathrm{Cd}^{2+} / \mathrm{Pb}^{2+}$ in the presence of aqueous environment. The authors assumed that such phenomena can be explained by the strong binding of $\mathrm{Hg}^{2+}$ to the surface functional groups (carboxyl and hydroxyl groups at the edges of GQD).More importantly, our results show that even in the absence of functional groups the optical signal from $\mathrm{Hg}^{2+} @$ GQD can be effectively quenched via the specific interaction of the GQD with $\mathrm{Hg}^{2+}$ in the presence of water. What is conclusive here is that GQD demonstrates high selectivity towards mercury ions, since the optical signal from GQD after applying the $\mathrm{Cd}^{2+}$ (these data were presented above) and $\mathrm{Pb}^{2+}$ (these data will be given below) ions in water remains unchanged compared to that of the pristine GQDs.

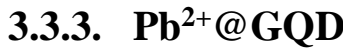

We now focus on the effect of the dielectric constant on the interaction strength between zigzag- and armchair-edged GQDs with $\mathrm{Pb}^{2+}$ ions. The binding energy of a $\mathrm{Pb}^{2+}$ ion can be tuned by controlling the dielectric constant of the solvent (Table 7). From energy point of view, the most favourable adsorption configuration is noted in the case when the $\mathrm{Pb}^{2+}$ ion sits in the hollow site of the planar structure of GQDs. The most preferred adsorption site remains the same even after complexation in polar solvents (see Table S8, Electronic Supplementary Information\#2), independently of the edge termination. It is worth mentioning that the charge transfer from GQDs to $\mathrm{Pb}^{2+}$ is quite low, even in vacuum conditions, in comparison to $\mathrm{Cd}^{2+}$ and $\mathrm{Hg}^{2+}$. Taking into account the fact that ionic radius of $\mathrm{Pb}^{2+}(119 \mathrm{pm})$ is much larger than that of $\mathrm{Cd}^{2+} / \mathrm{Hg}^{2+}$, it is reasonable to assume that the larger $\mathrm{Pb}^{2+}$ ion prefers to bind with GQDs in electrostatic manner. By this we mean that the small charge transfer observed in $\mathrm{Pb}^{2+} @ Z Z-G Q D$ and $\mathrm{Pb}^{2+} @ A C-G Q D$ is responsible for the reduced binding energy of $\mathrm{Pb}^{2+}$ in the gas-phase. Notwithstanding, we denoted that despite overall decrease in the interaction strength with increase in the dielectric constant the binding energies of $\mathrm{Pb}^{2+}$ in the solvated phases are higher than those of $\mathrm{Cd}^{2+} / \mathrm{Hg}^{2+}$. In fact, a change from the gas-phase to solvated phase causes the change of the binding sequence from $\mathrm{Hg}^{2+}>\mathrm{Cd}^{2+}>\mathrm{Pb}^{2+}$ to $\mathrm{Pb}^{2+}>\mathrm{Hg}^{2+}>\mathrm{Cd}^{2+}$, respectively. Similar reordering was also observed and explained for solvent-mediated interaction between metal cations and benzene ${ }^{58}$ and interaction of L-proline with group IIB $\left(\mathrm{Zn}^{2+}, \mathrm{Cd}^{2+}, \mathrm{Hg}^{2+}\right)$ metal cations ${ }^{57}$. 
Table 7. Computed parameters of zigzag- and armchair-edged GQDs after complexation with $\mathrm{Pb}^{2+}$ in different media

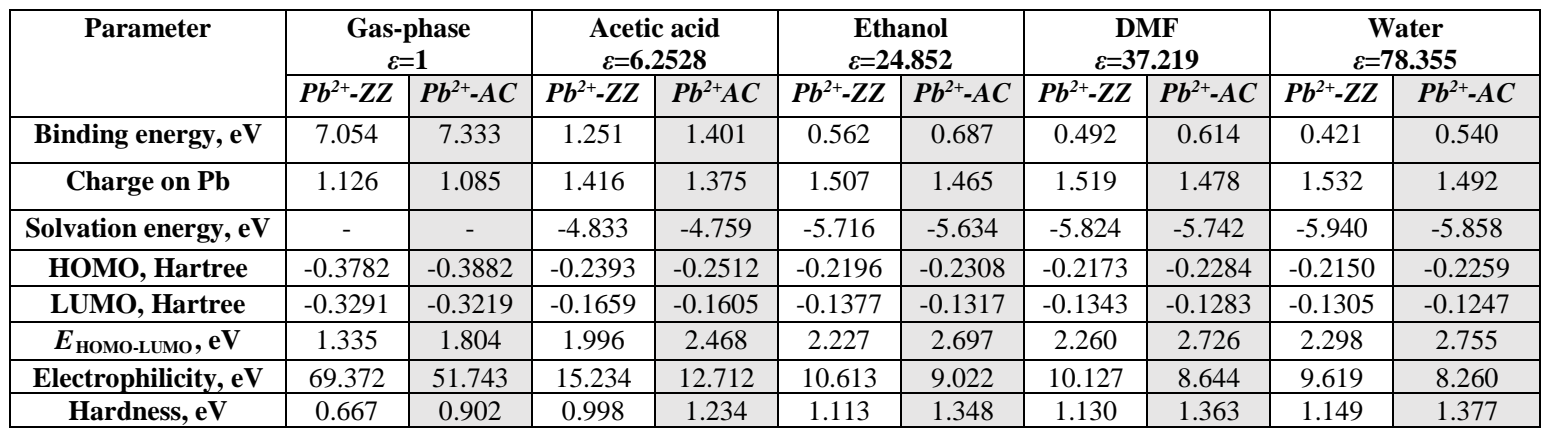

Several other aspects concerning the interaction between GQDs and divalent lead ion also deserve to be mentioned. First, the dependence of the electrophilicity index, global hardness and HOMO-LUMO gap energy on the dielectric permittivity of the solvent is, to a large extent, analogous to the typical dependences for $\mathrm{Hg}^{2+} @ G Q D$ and $\mathrm{Pb}^{2+} @ G Q D . J u s t$ as in those cases, the presence of the polar solvent makes the interacting system more stable and less reactive. This is evidenced by the HOMO-LUMO widening, the decrease in the electrophilicity index and an increase in global hardness. Note also that all mentioned parameters, while depending on the solvent effect, they are also very sensitive to the edge termination and shape of the GQD. In particular, $\mathrm{Pb}^{2+} @$ AC-GQDs immersed in different media are found to be more stable in comparison to $\mathrm{Pb}^{2+} @ Z Z-G Q D s$.

Bearing in mind the specific electronic properties of the $\mathrm{Pb}^{2+} @ Z Z-G Q D s$ and $\mathrm{Pb}^{2+} @ \mathrm{AC}-$ GQDs, we then consider their absorption spectra with the aim to understand the role of $\mathrm{Pb}^{2+}$ in the excited transitions. The absorption spectra of the considered systems immersed in different media are given in Fig. 14. As shown in Fig. 14 (left panel) and Fig. 15a, the absorption spectrum of $\mathrm{Pb}^{2+} @ Z Z-G Q D$ in the gas-phasecontains several very weak, barely resolved features at $595 \mathrm{~nm}, 1188 \mathrm{~nm}$ and $1383 \mathrm{~nm}$ (a full list of the allowed transitions can be found in Dataset S6, Electronic Supplementary Information\#2). The most intense component at $1188 \mathrm{~nm}$ with oscillator strength of 0.0085 can be ascribed to a HOMO$1 \rightarrow$ LUMO+1 transition. In this case, the wave function of the HOMO-1 level is delocalized over the planar structure of the GQD, while LUMO-1 is shared by GQD and $\mathrm{Pb}^{2+}$ (Figure S23, Electronic Supplementary Information\#2). The weak band at $595 \mathrm{~nm}$ is believed to arise from the combination of two electronic transitions: HOMO-3 $\rightarrow$ LUMO (69\%) and $\mathrm{HOMO} \rightarrow \mathrm{LUMO}+3(12 \%)$. According to the orbital composition analysis among these levels only LUMO is a hybrid orbital (ZZ-GQD: 17\%, Pb: 83\%).The lowest observed feature appears at $1383 \mathrm{~nm}$ and is originating from the following electronic transitions: HOMO- 
$1 \rightarrow$ LUMO $(11 \%)$ and $\mathrm{HOMO} \rightarrow \mathrm{LUMO}+1(88 \%)$. It should be mentioned that $\mathrm{Pb}^{2+} @ \mathrm{ZZ}-$ GQD in vacuum conditions has a twofold degenerate HOMO and a twofold degenerate LUMO and the solvent effect favours remaining of this degeneracy.Contrary to the gas-phase case, as shown in Fig. 14 (left panel), the acetic acid environment causes an obvious increase in the absorption intensity and the blue-shift of the absorption wavelengths (479 nm, $786 \mathrm{~nm}$ and $803 \mathrm{~nm}$ ). In principle, the nature of the observed absorption features are very similar to the gas-phase, including the electronic transitions between occupied orbitals(that related purely to GQD) and lowest unoccupied energy levels (hybridized in nature). Further increase in the dielectric constant of the solvent is accompanied by anincrease in the HOMO-LUMO energy gap. Due to this reason, the absorption spectrum undergoes an additional blue shiftfor solvents with the highest polarity (Fig. 15 c-e). Furthermore, we noticed a dramatic enhancement of the oscillator strengths for the electronic transitions in $\mathrm{Pb}^{2+} @ Z Z-G Q D$ dispersed in ethanol, DMF and water. Compared to the acetic acid, the intensive band ranging from 450 to $700 \mathrm{~nm}$ appears in the absorption spectra of the $\mathrm{Pb}^{2+} @ Z Z-G Q D$ in solvents with high polarity. As shown in Fig. 15c and Fig. 15d, the zigzag-edged GQD after complexation with $\mathrm{Pb}^{2+}$ in the presence of ethanol (or DMF) shows resolved peaks at 443 (443), 449 (445) and $456(454) \mathrm{nm}$, respectively. All these features are due to the transition from a $\pi$-orbital of GQD to a hybrid orbitals. The peak at $443 \mathrm{~nm}$ is mainly due to two overlapped transitions:HOMO-1 $\rightarrow$ LUMO+4 and $\mathrm{HOMO} \rightarrow \mathrm{LUMO}+2$. The feature at $449 \mathrm{~nm}$ corresponds to transition from HOMO-2 to LUMO, while the peak at $456 \mathrm{~nm}$ can be assigned to the following transitions: HOMO-2 $\rightarrow$ LUMO, HOMO- $\rightarrow$ LUMO +2 and $\mathrm{HOMO} \rightarrow \mathrm{LUMO}+4$. The highest blue-shift of the absorption spectra is observed in water (Figure 15e). In this case, $\mathrm{Pb}^{2+} @ Z Z-G Q D$ exhibits acombination of a very dense absorption bands at 438, 440 and 450 $\mathrm{nm}$, respectively. These features are mainly arising from the transition from $\pi$-symmetric GQD-based occupied molecular orbitals (degenerate HOMO and HOMO-2) to hybrid molecular orbitals (double degenerate LUMO/LUMO+1 and triple degenerate $\mathrm{LUMO}+2 / \mathrm{LUMO}+3 / \mathrm{LUMO}+4)$. The detailed description of all excited states is presented in Dataset S6(Electronic Supplementary Information\#2). To summarize the obtained results of solvent effect on the optical properties $\mathrm{Pb}^{2+} @ Z Z-G Q D$ we emphasize that the order of blueshifting is water $>$ DMF $>$ ethanol $>$ acetic acid $>$ vacuum.Our data also clearly show that the enhancement of absorption intensity follows the same trend. Therefore, it is reasonable to assume that solvent-induced weakening of the electrostatic interaction between GQD and $\mathrm{Pb}^{2+}$ favours the appearance of a strong absorption bands in visible. As the dielectric constant of the solvent increases, the density of unoccupied electronic states increases also and more 
allowed levels become available for occupation and thus can be involved in electronic transitions.Similar picture was observed for the armchair-edged GQDs interacted with $\mathrm{Pb}^{2+}$. Related discussions are described in Electronic Supplementary Information \#1 (Section 4).
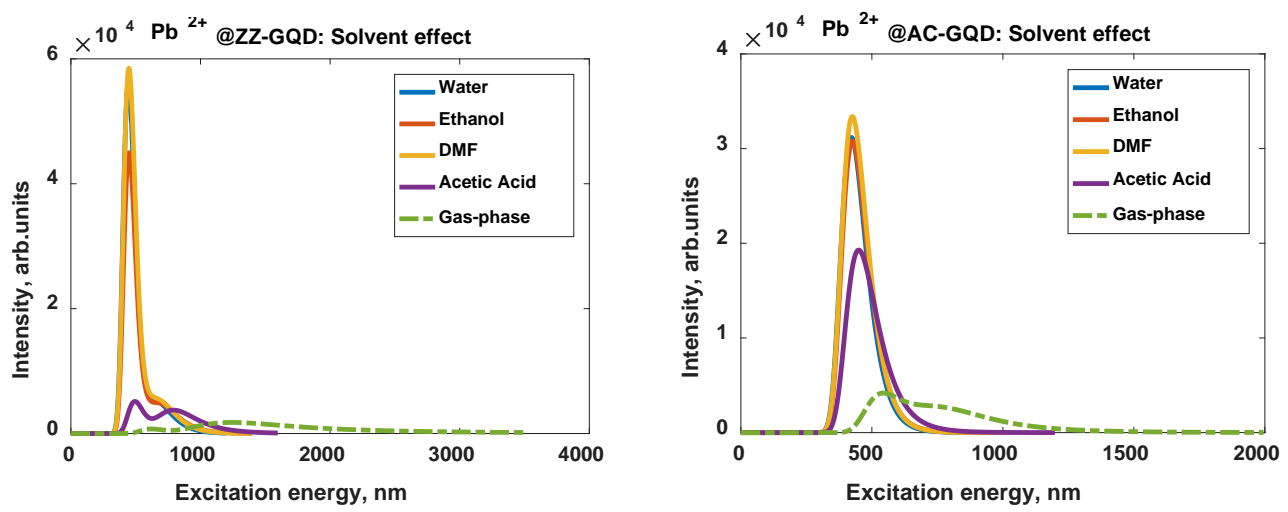

Figure 14. Absorption spectra of the GQDs after complexation with $\mathrm{Pb}^{2+}$ obtained in different dielectric media by using the PCM/TD-DFT/B3LYP/6-31G calculations of excited transitions: (left panel) zigzag edge termination and (right panel) armchair edge termination

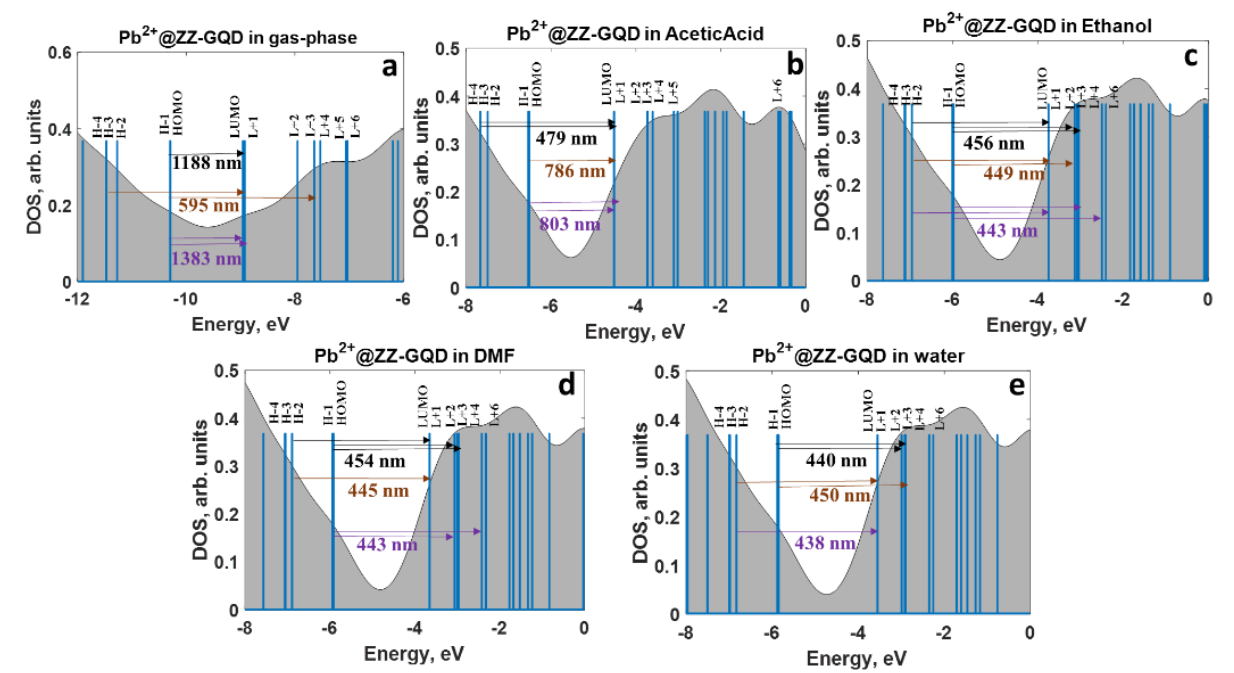

Figure 15. DOS of GQDs after complexation with $\mathrm{Pb}^{2+}$ (shaded area) immersed in different

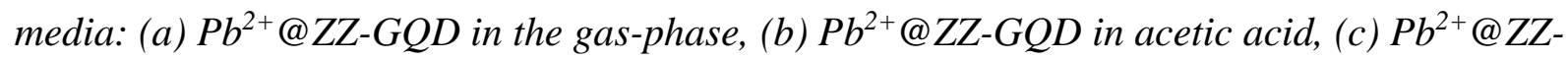
GQD in the ethanol, (d) $\mathrm{Pb}^{2+} @ Z Z-G Q D$ in the DMF, and (e) $P b^{2+} @ Z Z-G Q D$ in the water. Blue vertical lines denote the molecular orbitals. The excited transitions between available occupied and unoccupied electronic levels are shown by black arrowed lines.

\subsection{Role of the solvent-mediated interaction between HMs and GQDs in} electrochemical detection of the HMs

The aforementioned discussion illustrates mostly some important aspects of the solventcontrolled optical sensing of the heavy metals by the graphene quantum dots. Meanwhile, knowledge about the solvent-mediated interaction between heavy metals and differently 
shaped/sized GQDs can be also useful for elucidating the nature of the sensing mechanisms underlying the electrochemical detection of the HMs. Let us next to shed some light on how this method works. The electrochemical detection of metal ions starts from their adsorption on the surface of the electrode. Immediately after, the adsorbed ions are reduced to neutral species at a certain potential, and then the anodic stripping current related to the reoxidation of neutral metal atoms is measured to determine the amount of HM ions adsorbed on the electrode surface. Therefore, the main strategy to enhance the sensitivity of this method is to increase the adsorption capacity of the working electrode towards specified HM ions. In this regard, the GQDs can modify the surface of the typical working electrodes (glassy carbon, graphite, so on), thereby leading to improvement of the electrochemical performance. In other words, the role of GQDs is to enhance the anodic current density under stripping conditions via the improved interaction between the modified working electrode and metal ions. Here and further when we are discussing the electrochemical detection of the HMs using the modified electrode we mean that the surface modification of the working electrode is associated with two consequent preparation steps: (i) nano-sized GQDs should be initially dissolved into specific solvent and sonicated to prepare a uniform suspension and (ii) then the suspension can be dropped onto the surface of working electrode (for instance, GCE). After complete evaporation of the solvent at room temperature, the GQD-modified working electrode can be used in electrochemical cell.

Our DFT calculations suggest the different adsorption ability of the GQDs to heavy metal ions $\left(\mathrm{Cd}^{2+}, \mathrm{Hg}^{2+}\right.$ and $\left.\mathrm{Pb}^{2+}\right)$. This difference will be responsible for the different stripping behaviors for studied heavy metal ions. Keeping in mind the fact that the presence of the solvent weakens the interaction strength between GQDs and all HM ions in comparison to vacuum conditions (see Tables 5-7), we anticipate that the $\mathrm{Cd}^{2+}$ has the highest priority to be adsorbed on GQDs in the presence of the acetic acid (binding energy follows sequence $\mathrm{Cd}^{2+}>$ $\left.\mathrm{Hg}^{2+}>\mathrm{Pb}^{2+}\right)$. Thus, the acetic acid as a supporting electrolyte in electrochemical cell is expected to be more favorable medium for selective detection of the $\mathrm{Cd}^{2+}$ compared to others. On the other hand, we noticed that further increase in the dielectric permittivity of the medium (and $\mathrm{pH}$ of the solvent) leads to a rapid drop in the binding energy of $\mathrm{Cd}^{2+}$ and $\mathrm{Hg}^{2+}$ ions, while the binding energy of $\mathrm{Pb}^{2+}$ undergoes slower solvent-induced change. In particular, GQDs immersed in ethanol, DMF and/or water exhibit highest adsorption capacity toward $\mathrm{Pb}^{2+}$ than that toward other metal ions. This means that during reversible adsorption of the different metal ions onto modified electrode the selective preconcentration of $\mathrm{Pb}^{2+}$ at a working electrode will be the highest. When a negative potential is applied to the GQD- 
modified electrode more $\mathrm{Pb}^{2+}$ ions will be available to be plated as neutral $\mathrm{Pb}$ species on the electrode. As a result, the GQD modified electrode will exhibit higher stripping peak currents toward $\mathrm{Pb}^{2+}$. In fact, reliable controlling the solvent parameters (permittivity, content, $\mathrm{pH}$ ) is powerful tool to tune adsorption capacity, accumulation time of metal ions, preconcetration of the target HMs and anodic stripping peak current.Our findings show that the understanding of the nature of the solvent-mediated interaction between HM ions and GQDs is crucial for design of high-performance and highly-selective electrochemical sensor and for optimization of the conditions for the detection.

\section{Conclusions}

In summary, in order to gain deep insights into the nature of the excited states in HMs@GQDs we investigate the electronic and optical properties of the GQDs before and after complexation with neutral and divalent heavy metal species in the presence of different solvents (acetic acid, ethanol, DMF and water) by performing DFT and TD-DFT calculations. Our calculations of the ground state geometry indicate that the independently of the solvent effect and GQD edge termination the physisorped (weakly bounded) neutral Cd and $\mathrm{Hg}$ adatoms prefer to sit on the hollow site of the GQDs, while the Pb adatoms tend to bindvia the bridge site in a stronger manner. Most interestingly, the binding energy of the $\mathrm{Cd}$ and $\mathrm{Hg}$ slightly increased with increasing the dielectric constant of the solvent, while the binding energy of $\mathrm{Pb}$ is found to decrease. The weak interaction between $\mathrm{Cd} / \mathrm{Hg}$ and GQDs does not cause any important changes in the absorption spectra of the Cd@GQDs or Hg@GQDs in comparison to those of pristine GQDs. This is due to the fact that neither $\mathrm{Cd} / \mathrm{Hg}$-related molecular orbitals nor hybrid orbitals are found to be involved in electronic transitions for almost all cases, except the electronic transitions in Cd@AC-GQD in the gas-phase case. At the same time, we relate the strong quenching of the absorption spectrum of the GQDs after complexation with $\mathrm{Pb}$ to the both, dominating role of Pb-related HOMO level in excited transitions and solvent-induced shift of this level. In terms of sensing applications it means that GQD solution combined with UV-vis absorption spectroscopy technique can effectively detect only neutral $\mathrm{Pb}$ atoms, and is seem to be blind to $\mathrm{Cd}$ and $\mathrm{Hg}$.

It was also shown that the binding energy of the chemisorped divalent $\mathrm{Cd}^{2+}, \mathrm{Hg}^{2+}$ and $\mathrm{Pb}^{2+}$ ions is very sensitive to the dielectric permittivity of the solvent, demonstrating a dramatic decrease with increasing the solvent polarity. Solvent-mediated weakening of the interaction strength leads to a drastic change in the absorption properties of the HMs@GQDs. In particular, we foundthat the increase in the dielectric permittivity causes strengthening of the intensity of the transitions in relation to vacuum for $\mathrm{Cd}^{2+} @$ GQDs are Pb ${ }^{2+} @$ GQDs. In 
$\mathrm{Hg}^{2+} @ G Q D s$ the transitions between GQD-related doubly degenerate occupied and unoccupied energy levels are forbidden and only the Hg-related LUMO is involved in most likely transitions, which is believed to be a reason for the significant quenching of the absorption intensity in the presence of solvents with high polarity. Our results on optical sensing of the heavy metals ions are in good agreement with experimental findings and show the GQDs are a promising sensing materials for selective detection of $\mathrm{Hg}^{2+}$ ions in aqueous solutions. Furthermore, nanoscale GQDs are expected to be a promising materials for surface modification of the electrochemical working electrode. By choosing the proper electrolyte, it is possible to control the electrochemical performance of working electrode by tuning the adsorption capacity, accumulation time of metal ions, preconcetration of the target HMs.This study brings in fundamental understanding of the nature of the interaction between HMs and GQDs in the presence of solvents and elucidates the role of both the solvent and HM in electronic transitions. We believe that the precise control of the binding energy of the metal adsorbate on the nanographene surface and tuning the dielectric permittivity and $\mathrm{pH}$ of the solvent will provide the required background for the development of fast and real-time sensors for detection of HMs in waste water and bio-liquids.

\section{Acknowledgments}

The project has received funding from the European Union's Horizon 2020 research and innovation programme under grant agreement No 696656. The authors would like to thank financial support via VR grant 621-2014-5805 and SSF (SSF GMT14-0077, SSF RMA150024). Dr. I. Shtepliuk acknowledges the support from Wallenberg foundation and Ångpanneföreningens Forskningsstiftelse (Grant 16-541).

\section{References}

1 MSC-E Online, http://www.msceast.org/reports/2_2016.pdf, (accessed July 2016).

2 K.M. Rice, E. M. Walker, Jr, M. Wu, C. Gillette, and E. R. Blough, J. Prev. Med. Public Health, 2014, 47(2), 74-83.

3 N. Rifai, G. Cohen, M. Wolf, C. Faser, J. Savory, L. DePalma, Ther. Drug. Monit.,1993, 15, 71-74.

4 F. Depault, M. Cojocaru, F. Fortin, S. Chakrabarti, and N. Lemieux, Toxicology in Vitro, 2006, 20, 513-518.

5 A. C. Davis, P. Wu, X. Zhang, X. Hou, and B. T. Jones, Appl. Spectrosc. Rev., 2006, 41, 35-75.

6 N. H. Bings, A. Bogaerts, J. A. C. Broekaert,Anal. Chem. 2006, 78, 3917-3946.

7K. E. Lorber, Waste Manage. Res., 1986, 4, 3-13. 
8R. Kunkel, S. E. Manahan, Anal. Chem., 1973, 45, 1465-1468.

9M. Lopez-Artiguez, A. Camean, M. Repetto, J. Anal. Toxicol. 1993, 17, 18-22.

10 A. Ambrosi, C.K. Chua, A. Bonanni, M. Pumera, Chem. Rev., 2014,114, 7150-7188.

11 X. Wang, G. Sun, P. Routh, D.-H. Kim, W. Huang, P. Chen, Chem. Soc. Rev.,2014, 43, 7067-7098.

12 L. Li, G. Wu, G. Yang, J. Peng, J. Zhao, J.-J. Zhu, Nanoscale, 2013,5, 4015-4039.

13 X. Zheng, A. Ananthanarayanan, K. Qian Luo, P. Chen, Small, 2015, 11, 1620-1636.

14 X.T. Zheng, A. Than, A. Ananthanaraya, D.-H. Kim, P. Chen, ACS Nano, 2013, 7, 62786286.

15 H. Sun, L. Wu, W. Wei, X. Qu, Mater. Today, 2013, 16, 433-442.

16 C. Zhou, W. Jiang, B. K. Via,Colloids Surf., B, 2014, 118,72-76.

17Y.-X. Qi, M. Zhang, Q.-Q. Fu,R. Liu, G.-Y. Shi, Chem. Commun., 2013,49, 10599-10601.

18 M. A. Sk, A. Ananthanarayanan, L. Huang, K. H. Lim and P. Chen, J. Mater. Chem. C, 2014,2, 6954-6960.

19 I. Shtepliuk, N. M. Caffrey, T. Iakimov, V. Khranovskyy, I. A. Abrikosov and R. Yakimova,Sci. Rep., 2017, 7, 3934.

20 S. K. Pandey, P. Singh, J. Singh, S. Sachan, S. Srivastava, S. K. Singh,Electroanalysis, 2016, 28, 2472.

21 S.L. Ting, S. J. Ee, A. Ananthanarayanan, K. C. Leong, P. Chen,Electrochim. Acta, 2015, 172, 7-11.

22 H. Zhu, Y. Xu, A. Liu, N. Kong, F. Shan, W. Yang, C. J. Barrow, J. Liu,Sens. Actuators, B. 2015, 206, 592-600.

23 W. Lu, X. Qin, S. Liu, G. Chang, Y. Zhang, Y. Luo, A.M. Asiri, A.O. Al-Youbi, X. Sun, Anal. Chem., 2012, 84,5351-5357.

24H. Chakraborti, S. Sinha, S. Ghosh, S. K. Pal,Mater. Lett. 2013, 97, 78-80.

25 M. Park, H. D. Ha, Y. T. Kim, J. H. Jung, S.-H. Kim, D. H. Kim, T. S. Seo, Anal. Chem. 2015, 87, 10969-10975.

26 L. Li, G. Wu, G. Yang, J. Peng, J. Zhao and J. Zhu,Nanoscale, 2013, 5, 4015-4039.

27 D. Qu, M. Zheng, J. Li, Z. Xie and Z. Sun, Light: Sci. Appl., 2015,4, e364.

28 M. Zhao, F. Yang, Y. Xue, D. Xiao, and Y. Guo, ChemPhysChem, 2014, 15, 950 - 957.

29 X. Niu, Y. Li, H. Shua and J. Wang, Nanoscale, 2016, 8, 19376.

30M. J. Frisch, G. W. Trucks, H. B. Schlegel, G. E. Scuseria, M. A. Robb, J. R. Cheeseman, G. Scalmani, V. Barone, B. Mennucci, G. A. Petersson, H. Nakatsuji, M. Caricato,X. Li, H. P. Hratchian, A. F. Izmaylov, J. Bloino, G. Zheng, J. L. Sonnenberg, M. Hada, M. Ehara, K. 
Toyota, R. Fukuda, J. Hasegawa, M. Ishida, T. Nakajima, Y. Honda, O. Kitao, H. Nakai, T. Vreven, J. A. Montgomery,J. E. Peralta, F. Ogliaro, M. Bearpark, J. J. Heyd,E. Brothers, K. N. Kudin, V. N. Staroverov, R. Kobayashi,J. Normand, K. Raghavachari, A. Rendell, J. C. Burant, S. S. Iyengar, J. Tomasi, M. Cossi, N. Rega, J. M. Millam, M. Klene, J. E. Knox, J. B. Cross, V. Bakken, C. Adamo,J. Jaramillo, R. Gomperts, R. E. Stratmann, O. Yazyev,A. J. Austin, R. Cammi, C. Pomelli, J. W. Ochterski,R. L. Martin, K. Morokuma, V. G. Zakrzewski, G. A. Voth,P. Salvador, J. J. Dannenberg, S. Dapprich, A. D. Daniels,O. Farkas, J. B. Foresman, J. V. Ortiz, J. Cioslowski and D. J. Fox, Gaussian 09, Revision D.01, Gaussian Inc.,

Wallingford CT, 2009.

31 P. J. Stephens, F. J. Devlin, C. F. Chabalowski and M. J. Frisch, J. Phys. Chem., 1994, 98, 11623-11627.

32J. M. L. Martin, A. Sundermann, J. Chem. Phys., 2001, 114, 3408-3420.

33 M. Cossi, V. Barone, J. Chem. Phys. 2001, 115, 4708.

34S.F. Boys, F. Bernardi, Mol. Phys., 1970, 19, 553.

35 P. Geerlings, F. De Proft and W. Langenaeker, Chem. Rev., 2003, 103, 1793-1874.

36 P. K. Chattaraj, S. Giri and S. Duley, Chem. Rev., 2011, 2, PR43-PR75.

37 M. H. Cohen and A. Wasserman, J. Phys. Chem. A, 2007, 111, 2229-2242

38N. Winter, F. Ree, J. Comp. Mat. Des., 1998, 5, 279-294.

39 R. G. Parr, R. G. Pearson, J. Am. Chem. Soc., 1983, 105, 7512 - 7516.

40 M. Shakourian-Fard, Z. Jamshidi, G.Kamath, ChemPhysChem, 2016, 17, 3289 - 3299.

41 A. Klamt and V. Jonas, J. Chem. Phys., 1996, 105, 9972- 9981.

42 A. Klamt and G. Schüürmann, J. Chem. Soc., Perkin Trans.,1993, 2, 799-805.

43 C. C. Pye and T. Ziegler, Theor. Chem. Acc., 1999, 101, 396- 408.

44M. Legesse, F.E. Mellouhi, E. T. Bentria, M. E. Madjet, T. S Fisher, S. Kais, F. H Alharbi, Appl. Surf. Sci.,2017, 394, 98-107.

45W. C. Martin, A. Musgrove, S. Kotochigova, Ground Levels and Ionization Energies for Neutral Atoms, NIST, Gaithersburg, MD, 2002.

46D. Lide, CRC Handbook of Chemistry and Physics,CRC Press, Boca Raton, FL, 2005.

47 M. Darvish Ganji, S. M. Hosseini-khah and Z. Amini-tabar, Phys. Chem. Chem. Phys., 2015, 17, 2504.

48 T. Yumura, T. Awano, H. Kobayashi and T. Yamabe, Molecules, 2012, 17, 7941-7960.

49 V. S. Anithaa, R. Shankar, S. Vijayakumar, J. Mol. Model., 2017, 23, 132.

50 Y. Zhao, D. G. Truhlar, J. Chem. Phys., 2006, 125, 194101. 
51 Y. Zhao, D. G. Truhlar, Theor. Chem. Acc., 2008, 120, 215-241.

52 J. P. Perdew, K. Burke, M. Ernzerhof, Phys. Rev. Lett., 1996, 77, 3865.

53 J. P. Gallivan and D. A. Dougherty, J. Am. Chem. Soc., 2000, 122, 870-874

54J. S. Rao, H. Zipse, G. N. Sastry, J. Phys. Chem. B, 2009, 113, 7225-7236.

55G. J. Fleming, P. R. McGill, H. Idriss, J. Phys. Org. Chem., 2007, 20, 1032.

56M. Belcastro, T. Marino, N. Russo, M. Toscano, J. Mass Spectrom., 2005, 40, 300

57 B. Khalili, M. Rimaz, Can. J. Chem., 2016, 94, 501-50.

58 R. Kumpf, D. Dougherty, Science, 1993,261, 1708-10. 